\title{
Non-topological Vortex Configurations in the ABJM Model*
}

\author{
Xiaosen $\operatorname{Han}^{a, b} \quad$ Gabriella Tarantello ${ }^{a}$ \\ ${ }^{a}$ Dipartimento di Matematica, Universita degli Studi di Roma Tor Vergatà, \\ Via della Ricerca Scientifica, 00133 Rome, Italy \\ ${ }^{b}$ Institute of Contemporary Mathematics, School of Mathematics, Henan University \\ Kaifeng 475004, PR China
}

\begin{abstract}
In this paper we study the existence of vortex-type solutions for a system of self-dual equations deduced from the mass-deformed Aharony-Bergman-Jafferis-Maldacena (ABJM) model. The governing equations, derived by Mohammed, Murugan, and Nastse under suitable ansatz involving fuzzy sphere matrices, have the new feature that they can support only non-topological vortex solutions. After transforming the self-dual equations into a nonlinear elliptic $2 \times 2$ system we prove first an existence result by means of a perturbation argument based on a new and appropriate scaling for the solutions. Subsequently, we prove a more complete existence result by using a dynamical analysis together with a blow-up argument. In this way we establish that, any positive energy level is attained by a 1-parameter family of vortex solutions which also correspond to (constraint) energy minimizers. In other words, we register the exceptional fact in a BPS-setting that, neither a "quantization" effect nor an energy gap is induced upon the system by the rigid "critical" coupling of the self-dual regime.
\end{abstract}

Key words: ABJM model, self-dual vortex equations, non-topological vortices, nonlinear elliptic equations

Mathematics subject classifications (2010): 35J47, 35J60, 81T60

\section{Introduction}

Abelian and non-Abelian Chern-Simons vortices have been studied in a series of relativistic or non-relativistic field models [17, 28, 31, 36, 37, 54. These configurations play important roles in both theoretical and experimental studies in modern physics [16, 20, 33, 41, 58, 59, 62. Recently the Chern-Simons action has received much attention in connection with Superstring theory and Mtheory [57. Based on the seminal work of Bagger-Lambert [4 6] and Gustavsson [23] (BLG), in [1] Aharony, Bergman, Jafferis and Maldacena (ABJM) have constructed an $\mathcal{N}=6$ superconformal $U(N) \times U(N)$ Chern-Simons gauge theory with level $(k,-k)$, coupled to four complex scalars and four fermions in the bifundamental representation. This theory describes the world-volume dynamics of $N$ coincident $M 2$-branes moving in a $\mathbb{C}_{4} / \mathbb{Z}_{k}$ orbifold background in M-theory.

*This work is supported by PRIN12: "Variational and Perturbative Aspects in Nonlinear Differential Problems" and by FIRB project: "Analysis and Beyond". 
For both models (BLG and ABJM), it is interesting to identify soliton-like objects, such as domain walls, vortices, and Q-balls, since they correspond to various configurations of membranes. To this purpose, in [34] the authors derived self-dual equations of BPS-type [8, 56] for the massdeformed ABJM model with gauge group $U(N) \times U(N)$. When $N=2$ such BPS equations reduce to the Abelian Higgs equation [22, 30, 50] whose existence theory is completely understood [30]. While for $N>2$, the BPS equations give rise to more general systems of the type describing non-Abelian vortex configurations, for which existence and uniqueness results were established recently in [27. We mention also [3], where another set of BPS equations were derived in the massdeformed ABJM model at both weak and strong coupling, in this case existence and uniqueness results were established in [38]. For more recent developments concerning the ABJM model see [2, 7, 29, 35, 40, 44, 47, 52, 53, and the references therein.

In order to find possible applications of the ABJM theory to specific condensed matter systems, recently a consistent Abelian truncations of the mass-deformed ABJM model was introduced by Mohammed-Murugan-Nastse [42,43]. By means of suitable ansatz involving fuzzy sphere matrices, the authors of [42,43] were able to reduce the model into an effective Abelian field model, which admits a BPS reduction. However, due to the specific ansatz introduced in [42,43], the corresponding system of self-dual equations admits new features compared to those obtained in [3, 34]. In particular, we see that, to attain finite energy, the corresponding self-dual vortex configurations can be only of non-topological type. This is a surprising new feature, when compared to more familiar BPS equations arising both in Abelian and non-Abelian contexts also in presence of the Chern-Simons terms, see [12,17, 19,24, 26, 28, 31, 36, 39, 54,60,63] and references therein. Even more importantly, for the BPS system in [43] we observe no energy-gap featuring along its solutions, as in fact each (positive) energy level is always attained by a (constraint) energy minimizer. The main purpose of this paper is to establish with mathematical rigor all such new features characterizing the Abelian truncation of the mass-deformed ABJM model proposed in [42, 43]. Thus, we shall prove existence and multiplicity results for the system of BPS equations in [43] in terms of the corresponding energy and fluxes. As usual, to tackle such issues, we reduce the self-dual equations into a planar $2 \times 2$ system of nonlinear elliptic equations, except that now we face an "indefinite" system of elliptic PDE's, particularly delicate to handle by the available analytical and variational tools. Indeed, so far we are aware only of the numerical study provided in [43].

We shall focus to the case where all vortex points are superimposed (with assigned multiplicity) at a given point, and thus we search for radially symmetric solutions about such point. We point out that even the radial solvability of the given system of PDE's is non-trivial. In fact, we lack the standard a priori estimates, so that not all entire radial solutions satisfying non-topological boundary conditions give rise to finite-energy configurations. However, we manage to show that some partial integrability can be always guaranteed. In addition, we shall construct a first class of finite-energy (radial) solutions, which enjoy a physically interesting "concentration" property. To this end, we use a perturbation approach introduced in [9] to deal with Abelian non-topological Chern-Simons vortices, and further developed in [10,11] for self-dual electroweak models with and without gravitational effects. More precisely, we shall identify an appropriate new scaling for the solution, which, in one hand, sets our problem into a "perturbation" framework, and at the same time also helps to clarify its specific analytic features. With this information in hand, and by means of a blow-up analysis, we arrive to identify necessary and sufficient conditions, which characterize 
the sharp range attained by the total energy (or by the two fluxes) along BPS-solutions. In fact, for each value in this range, we construct a 1-parameter family of solutions carrying such fixed amount of total energy or flux. This indicates that, for the given model, the set of total fluxes covers a planar region bounded by two curves emanating from a zero energy configuration. See Theorem 2.2 and 2.3 for the precise statements. In this way, we record the absence of "quantization" effects as well as energy gaps along BPS solutions.

The rest of this paper is organized as follows. In Section 2, (following [43]) we review the ABJM model, the associated equations of motion, the Abelian truncation and the derivation of the selfdual equations, and state our existence results. In Section 3 we present necessary conditions for the existence of finite-energy self-dual solutions. To show their existence, in section 4 we introduce a new scaling, which enables us to view our problem as a perturbation of a Liouville system. So we shall apply the perturbation approach introduced in [9] to construct a class of radially symmetric solutions, which however do not exhaust the full range of admissible energies (or fluxes). Thus, to complete our analysis, we provide in Section 5 some general information about radial solutions, and in particular we analyze the Cauchy problem and realize that, the finite-energy conditions may not be always attained. Therefore, by means of a blow-up analysis in Section 6, we shall be able to identify suitable set of initial data which yield to the desired 1-parameter family of finite-energy solutions.

\section{Self-dual equations and existence theorems}

Let $g_{\mu \nu}=\operatorname{diag}(+1,+1,-1)$ be the metric in the Minkowski spacetime $\mathbb{R}^{2,1}$. In the following we use (unless otherwise specified) the standard notation of summation over repeated lower and upper indices, and where indices are raised or lowered by using the metric in the usual way. The ABJM model [1] is an $\mathcal{N}=6$ supersymmetric $U(N) \times U(N)$ Chern-Simons gauge theory with level $(k,-k)$ coupled to four complex scalars $C^{I}$ and four Dirac fermions $\psi_{I}$ in the fundamental representation of the $S U(4)_{R}$ symmetry group, where the R-symmetry index $I=1, \ldots, 4$. Also, we denote the gauge fields for the two groups respectively $A_{\mu}$ and $\hat{A}_{\mu}$, with Lorentz index $\mu=0,1,2$. The ABJM action is defined as follows:

$$
\mathcal{A}=\int\left(\mathcal{L}_{\mathrm{CS}}+\mathcal{L}_{\mathrm{kin}}-V_{\text {ferm }}-V_{0}\right) \mathrm{d} x
$$

where $\mathcal{L}_{\mathrm{CS}}$ denotes the Chern-Simons Lagrangian density:

$$
\mathcal{L}_{\mathrm{CS}}=\frac{k}{4 \pi} \epsilon^{\mu \nu \lambda} \operatorname{Tr}\left(A_{\mu} \partial_{\nu} A_{\lambda}+\frac{2 i}{3} A_{\mu} A_{\nu} A_{\lambda}-\hat{A}_{\mu} \partial_{\nu} \hat{A}_{\lambda}-\frac{2 i}{3} \hat{A}_{\mu} \hat{A}_{\nu} \hat{A}_{\lambda}\right)
$$

and the matter-kinetic Lagrangian density $\mathcal{L}_{\text {kin }}$ is given by:

$$
\mathcal{L}_{\text {kin }}=-\operatorname{Tr}\left(D_{\mu} C_{I}^{\dagger} D^{\mu} C^{I}\right)-i \operatorname{Tr}\left(\psi^{I \dagger} \gamma^{\mu} D_{\mu} \psi_{I}\right),
$$

with $\epsilon^{\mu \nu \lambda}$ the antisymmetric Levi-Civita tensor fixed by setting $\epsilon^{012}=1$, and $k>0$ the ChernSimons level. In addition, the Dirac matrices $\gamma^{\mu}$ are expressed in terms of the standard Pauli spin matrices, $\gamma^{0}=\mathrm{i} \sigma^{2}, \gamma^{1}=\sigma^{1}, \gamma^{2}=\sigma^{3}$, and the gauge-covariant derivatives are defined by:

$$
\begin{aligned}
D_{\mu} C^{I} & =\partial_{\mu} C^{I}+\mathrm{i}\left(A_{\mu} C^{I}-C^{I} \hat{A}_{\mu}\right), \\
D_{\mu} \psi_{I} & =\partial_{\mu} \psi_{I}+\mathrm{i}\left(A_{\mu} \psi_{I}-\psi_{I} \hat{A}_{\mu}\right) .
\end{aligned}
$$


Furthermore, in (2.1), $V_{\text {ferm }}$ is the Yukawa-type quartic-interaction potential density given by:

$$
\begin{aligned}
V_{\text {ferm }}= & \frac{2 \pi i}{k} \operatorname{Tr}\left(C_{I}^{\dagger} C^{I} \psi^{J \dagger} \psi_{J}-\psi^{J \dagger} C^{I} C_{I}^{\dagger} \psi_{J}-2 C_{I}^{\dagger} C^{J} \psi^{I \dagger} \psi_{J}+2 \psi^{J \dagger} C^{I} C_{J}^{\dagger} \psi_{J}\right. \\
& \left.+\epsilon^{I J K L} C_{I}^{\dagger} \psi_{J} C_{K}^{\dagger} \psi_{L}-\epsilon_{I J K L} C^{I} \psi^{J \dagger} C^{K} \psi^{L \dagger}\right) ;
\end{aligned}
$$

and $V_{0}$ is the sextic scalar potential:

$$
\begin{aligned}
V_{0}= & \frac{4 \pi^{2}}{3 k^{2}} \operatorname{Tr}\left(C^{I} C_{I}^{\dagger} C^{J} C_{J}^{\dagger} C^{K} C_{K}^{\dagger}+C_{I}^{\dagger} C^{I} C_{J}^{\dagger} C^{J} C_{K}^{\dagger} C^{K}+4 C^{I} C_{J}^{\dagger} C^{K} C_{I}^{\dagger} C^{J} C_{K}^{\dagger}\right. \\
& \left.-6 C^{I} C_{J}^{\dagger} C^{J} C_{I}^{\dagger} C^{K} C_{K}^{\dagger} C^{K}\right),
\end{aligned}
$$

where again: $\epsilon^{1234}=1$, and the antisymmetric tensor $\epsilon^{I J K L}$ takes values \pm 1 according to the whether the set of indices $\{I J K L\}$ is an even or odd permutation of $\{1234\}$ and it is set to zero otherwise.

The ABJM action exhibits an $S U(4) \times U(1)$ R-symmetry associated with the $\mathcal{N}=6$ supersymmetric transformation [1, 34. This theory has a maximally supersymmetric massive deformation with mass parameter $\sigma$ [21, 61, which breaks the R-symmetry down to $S U(2) \times S U(2) \times U(1)_{A} \times$ $U(1)_{\hat{A}} \times \mathbb{Z}_{2}$ by decomposing the scalars as follows:

$$
C^{I}=\left(Q^{\alpha}, R^{\alpha}\right), \quad \alpha=1,2 .
$$

In this way the $\mathbb{Z}_{2}$-action swaps the matter fields $Q^{\alpha}$ and $R^{\alpha}$, the $S U(2)$-action is taken individually on $Q^{\alpha}$ and $R^{\alpha}$ respectively, while the $U(1)$ symmetry rotates $Q^{\alpha}$ on phase +1 and $R^{\alpha}$ by a phase -1 . The bosonic part of the mass-deformed Lagrangian takes the form [42, 43]:

$$
\begin{aligned}
\mathcal{L}_{\text {Bosonic }} & =\frac{k}{4 \pi} \epsilon^{\mu \nu \lambda} \operatorname{Tr}\left(A_{\mu} \partial_{\nu} A_{\lambda}+\frac{2 \mathrm{i}}{3} A_{\mu} A_{\nu} A_{\lambda}-\hat{A}_{\mu} \partial_{\nu} \hat{A}_{\lambda}-\frac{2 \mathrm{i}}{3} \hat{A}_{\mu} \hat{A}_{\nu} \hat{A}_{\lambda}\right) \\
& -\operatorname{Tr}\left(\left|D^{\mu} Q^{\alpha}\right|^{2}\right)-\operatorname{Tr}\left(\left|D^{\mu} R^{\alpha}\right|^{2}\right)-V,
\end{aligned}
$$

in which the potential $V$ takes the following sextic form:

$$
V=\operatorname{Tr}\left(\left|M^{\alpha}\right|^{2}+\left|N^{\alpha}\right|^{2}\right)
$$

with

$$
\begin{gathered}
M^{\alpha}=\sigma Q^{\alpha}+\frac{2 \pi}{k}\left(2 Q^{[\alpha} Q_{\beta}^{\dagger} Q^{\beta]}+R^{\beta} R_{\beta}^{\dagger} Q^{\alpha}-Q^{\alpha} R_{\beta}^{\dagger} R^{\beta}+2 Q^{\beta} R_{\beta}^{\dagger} R^{\alpha}-2 R^{\alpha} R_{\beta}^{\dagger} Q^{\beta}\right), \\
N^{\alpha}=-\sigma R^{\alpha}+\frac{2 \pi}{k}\left(2 R^{[\alpha} R_{\beta}^{\dagger} R^{\beta]}+Q^{\beta} Q_{\beta}^{\dagger} R^{\alpha}-R^{\alpha} Q_{\beta}^{\dagger} Q^{\beta}+2 R^{\beta} Q_{\beta}^{\dagger} Q^{\alpha}-2 Q^{\alpha} Q_{\beta}^{\dagger} R^{\beta}\right),
\end{gathered}
$$

and where the notation $Y^{[\alpha} Y_{\gamma}^{\dagger} Y^{\beta]} \equiv\left(Y^{\alpha} Y_{\gamma}^{\dagger} Y^{\beta}-Y^{\beta} Y_{\gamma}^{\dagger} Y^{\alpha}\right)$ is observed.

The Euler-Lagrange equations of the bosonic Lagrangian (2.9) are given by [42,43]:

$$
\begin{aligned}
\frac{k}{2 \pi} \epsilon^{\mu \nu \lambda} F_{\nu \lambda} & =J^{\mu}, \\
\frac{k}{2 \pi} \epsilon^{\mu \nu \lambda} \hat{F}_{\nu \lambda} & =\hat{J}^{\mu}, \\
D_{\mu} D^{\mu} Q^{\alpha} & =\frac{\partial V}{\partial Q_{\alpha}^{\dagger}}, \\
D_{\mu} D^{\mu} R^{\alpha} & =\frac{\partial V}{\partial R_{\alpha}^{\dagger}},
\end{aligned}
$$


with field strengths:

$$
F_{\mu \nu}=\partial_{\mu} A_{\nu}-\partial_{\nu} A_{\mu}+\mathrm{i}\left[A_{\mu}, A_{\nu}\right], \quad \hat{F}_{\mu \nu}=\partial_{\mu} \hat{A}_{\nu}-\partial_{\nu} \hat{A}_{\mu}+\mathrm{i}\left[\hat{A}_{\mu}, \hat{A}_{\nu}\right],
$$

and the two currents $J^{\mu}$ and $\hat{J}^{\mu}$ defined by

$$
\begin{aligned}
& J^{\mu} \equiv \mathrm{i}\left(Q^{\alpha}\left(D^{\mu} Q^{\alpha}\right)^{\dagger}-\left(D^{\mu} Q^{\alpha}\right) Q_{\alpha}^{\dagger}+R^{\alpha}\left(D^{\mu} R^{\alpha}\right)^{\dagger}-\left(D^{\mu} R^{\alpha}\right) R_{\alpha}^{\dagger}\right) \\
& \hat{J}^{\mu} \equiv-\mathrm{i}\left(Q_{\alpha}^{\dagger}\left(D^{\mu} Q^{\alpha}\right)-\left(D^{\mu} Q^{\alpha}\right)^{\dagger} Q^{\alpha}+R_{\alpha}^{\dagger}\left(D^{\mu} R^{\alpha}\right)-\left(D^{\mu} R^{\alpha}\right)^{\dagger} R^{\alpha}\right)
\end{aligned}
$$

which are covariantly conserved: $\nabla_{\mu} J^{\mu}=\nabla_{\mu} \hat{J}^{\mu}=0$. In addition, there are two ordinarily conserved Abelian currents $j^{\mu}$ and $\hat{j}^{\mu}$ :

$$
\begin{aligned}
& j^{\mu} \equiv \mathrm{i} \operatorname{Tr}\left(Q^{\alpha}\left(D^{\mu} Q^{\alpha}\right)^{\dagger}-\left(D^{\mu} Q^{\alpha}\right) Q_{\alpha}^{\dagger}+R^{\alpha}\left(D^{\mu} R^{\alpha}\right)^{\dagger}-\left(D^{\mu} R^{\alpha}\right) R_{\alpha}^{\dagger}\right) \\
& \hat{j}^{\mu} \equiv-\mathrm{i} \operatorname{Tr}\left(Q_{\alpha}^{\dagger}\left(D^{\mu} Q^{\alpha}\right)-\left(D^{\mu} Q^{\alpha}\right)^{\dagger} Q^{\alpha}+R_{\alpha}^{\dagger}\left(D^{\mu} R^{\alpha}\right)-\left(D^{\mu} R^{\alpha}\right)^{\dagger} R^{\alpha}\right)
\end{aligned}
$$

with respect to the global $U(1)_{A}$ and $U(1)_{\hat{A}}$ invariances.

In view of the complicated structures of the equations (2.13)-(2.16), one is prompted to consider some simplification. More precisely, by introducing the real-valued vector fields $a_{\mu}^{(1)}$ and $a_{\mu}^{(2)}$, and the complex-valued scalar fields $\phi_{\alpha}$ and $\chi_{\alpha}$, Mohammed-Murugan-Nastase [42, 43] proposed the following Abelianization ansatz for the Chern-Simons fields and the matter scalars:

$$
\begin{aligned}
A_{\mu} & =a_{\mu}^{(2)} G^{1} G_{1}^{\dagger}+a_{\mu}^{(1)} G^{2} G_{2}^{\dagger}, \\
\hat{A}_{\mu} & =a_{\mu}^{(2)} G_{1}^{\dagger} G^{1}+a_{\mu}^{(1)} G_{2}^{\dagger} G^{2}, \\
Q^{\alpha} & =\phi_{\alpha} G^{\alpha} \\
R^{\alpha} & =\chi_{\alpha} G^{\alpha}
\end{aligned}
$$

$\alpha=1,2$ and $\mu=0,1,2$ (no summation over $\alpha$ in $(2.24)-(2.25)$ ), with $G^{\alpha}$ defined as follows:

$$
\begin{aligned}
\left(G^{1}\right)_{m, n}=\sqrt{m-1} \delta_{m, n}, & \left(G^{2}\right)_{m, n}=\sqrt{N-m} \delta_{m+1, n}, \\
\left(G_{1}^{\dagger}\right)_{m, n}=\sqrt{m-1} \delta_{m, n}, & \left(G_{2}^{\dagger}\right)_{m, n}=\sqrt{N-n} \delta_{n+1, m},
\end{aligned}
$$

$m, n \in\{1, \ldots, N\}$. In particular, the mass-deformed theory admits the following ground state of the fuzzy type [21,61]:

$$
R^{\alpha}=c G^{\alpha}, \quad Q^{\alpha}=0 \quad \text { and } \quad Q_{\alpha}^{\dagger}=c G^{\alpha}, \quad R^{\alpha}=0,
$$

with

$$
c \equiv \sqrt{\frac{\sigma k}{2 \pi}} .
$$

Actually, the ground state (2.28) corresponds to a fuzzy 2-sphere [48,49]. Note also that the matrices $G^{\alpha}$, bifundamental under $U(N) \times U(N)$, satisfy:

$$
G^{\alpha}=G^{\alpha} G_{\beta}^{\dagger} G^{\beta}-G^{\beta} G_{\beta}^{\dagger} G^{\alpha}, \quad \alpha, \beta=1,2,
$$

(again no summation on repeated indices in (2.30) $)$. 
The above ansatz leads to a consistent Abelian truncation of the ABJM Lagrangian [42,43,

$$
\mathcal{L}_{\mathrm{td}}=-\frac{N(N-1)}{2}\left[\frac{k}{4 \pi} \epsilon^{\mu \nu \lambda}\left(a_{\mu}^{(2)} f_{\nu \lambda}^{(1)}+a_{\mu}^{(1)} f_{\nu \lambda}^{(2)}\right)+\left|D_{\mu} \phi_{i}\right|^{2}+\left|D_{\mu} \chi_{i}\right|^{2}+U\left(\left|\phi_{i}\right|,\left|\chi_{i}\right|\right)\right]
$$

with the following reduced potential $U \equiv 2 V / N(N-1)$ :

$$
\begin{aligned}
U= & \frac{4 \pi^{2}}{k^{2}}\left[\left(\left|\phi_{1}\right|^{2}+\left|\chi_{1}\right|^{2}\right)\left(\left|\chi_{2}\right|^{2}-\left|\phi_{2}\right|^{2}-c^{2}\right)^{2}+4\left|\phi_{1}\right|^{2}\left|\phi_{2}\right|^{2}\left(\left|\chi_{1}\right|^{2}+\left|\chi_{2}\right|^{2}\right)\right. \\
& \left.+\left(\left|\phi_{2}\right|^{2}+\left|\chi_{2}\right|^{2}\right)\left(\left|\chi_{1}\right|^{2}-\left|\phi_{1}\right|^{2}-c^{2}\right)^{2}+4\left|\chi_{1}\right|^{2}\left|\chi_{2}\right|^{2}\left(\left|\phi_{1}\right|^{2}+\left|\phi_{2}\right|^{2}\right)\right]
\end{aligned}
$$

Clearly, the induced Abelian gauge covariant derivatives are given by:

$$
D_{\mu} \phi_{i}=\partial_{\mu} \phi_{i}-\mathrm{i} a_{\mu}^{(i)} \phi_{i}, \quad D_{\mu} \chi_{i}=\partial_{\mu} \chi_{i}-\mathrm{i} a_{\mu}^{(i)} \chi_{i}
$$

with the induced Abelian field strengths:

$$
f_{\mu \nu}^{(i)}=\partial_{\mu} a_{\nu}^{(i)}-\partial_{\nu} a_{\mu}^{(i)}, \quad i=1,2 .
$$

The above Abelianization involves four complex-valued scalars, and this general situation can be simplified further by setting: $\chi_{1}=\phi_{2}=0$. Then, with abuse of notation, where one denotes $\phi_{2}$ in place of $\chi_{2}$, in [42, 43] Mohammed, Murugan and Nastase obtained the following effective Lagrangian:

$$
\mathcal{L}_{\mathrm{td}}=-\frac{N(N-1)}{2}\left[\frac{k}{4 \pi} \epsilon^{\mu \nu \lambda}\left(a_{\mu}^{(2)} f_{\nu \lambda}^{(1)}+a_{\mu}^{(1)} f_{\nu \lambda}^{(2)}\right)+\left|D_{\mu} \phi_{i}\right|^{2}+U\left(\left|\phi_{1}\right|,\left|\phi_{2}\right|\right)\right],
$$

with

$$
U\left(\left|\phi_{1}\right|,\left|\phi_{2}\right|\right)=\frac{4 \pi^{2}}{k^{2}}\left[\left|\phi_{1}\right|^{2}\left(\left|\phi_{2}\right|^{2}-c^{2}\right)^{2}+\left|\phi_{2}\right|^{2}\left(\left|\phi_{1}\right|^{2}+c^{2}\right)^{2}\right]
$$

and $c$ given in (2.29).

It can be easily checked that the equations of motion for the truncated Lagrangian (2.35) take the form:

$$
\begin{aligned}
\frac{k}{2 \pi} \epsilon^{\mu \nu \alpha} f_{\nu \alpha}^{(1)} & =\mathrm{i}\left(\phi_{2} \overline{D^{\mu} \phi_{2}}-\overline{\phi_{2}} D^{\mu} \phi_{2}\right), \\
\frac{k}{2 \pi} \epsilon^{\mu \nu \alpha} f_{\nu \alpha}^{(2)} & =\mathrm{i}\left(\phi_{1} \overline{D^{\mu} \phi_{1}}-\overline{\phi_{1}} D^{\mu} \phi_{1}\right), \\
D_{\mu} D^{\mu} \phi_{1} & =\frac{4 \pi^{2}}{k^{2}}\left[\left(\left|\phi_{2}\right|^{2}-c^{2}\right)^{2}+2\left|\phi_{2}\right|^{2}\left(\left|\phi_{1}\right|^{2}+c^{2}\right)\right] \phi_{1}, \\
D_{\mu} D^{\mu} \phi_{2} & =\frac{4 \pi^{2}}{k^{2}}\left[2\left|\phi_{1}\right|^{2}\left(\left|\phi_{2}\right|^{2}-c^{2}\right)+\left(\left|\phi_{1}\right|^{2}+c^{2}\right)^{2}\right] \phi_{2} .
\end{aligned}
$$

We are interested to obtain static solutions of (2.37)-(2.40). To this purpose, we use the Gauss laws of the Lagrangian (2.35) (expressed by the $\mu=0$ component of (2.37)-(2.38) $)$, given as follows:

$$
\begin{aligned}
\frac{k}{2 \pi} f_{12}^{(1)} & =a_{0}^{(2)}\left|\phi_{2}\right|^{2}, \\
\frac{k}{2 \pi} f_{12}^{(2)} & =a_{0}^{(1)}\left|\phi_{1}\right|^{2},
\end{aligned}
$$


together with the following well-known identities:

$$
\left|D_{1} \phi_{i}\right|^{2}+\left|D_{2} \phi_{i}\right|^{2}=\left|D_{1} \phi_{i} \pm \mathrm{i} D_{2} \phi_{i}\right|^{2} \pm \mathrm{i}\left(\partial_{1}\left[\phi_{i} \overline{D_{2} \phi_{i}}\right]-\partial_{2}\left[\phi_{i} \overline{D_{1} \phi_{i}}\right]\right) \pm f_{12}^{(i)}\left|\phi_{i}\right|^{2}, i=1,2
$$

As a consequence, we can write the static energy density relative to (2.35) as follows:

$$
\begin{aligned}
\mathcal{E}= & -\mathcal{L}_{\mathrm{td}}=\frac{N(N-1)}{2}\left\{\left(a_{0}^{(1)}\right)^{2}\left|\phi_{1}\right|^{2}+\left(a_{0}^{(2)}\right)^{2}\left|\phi_{2}\right|^{2}+\sum_{i=1}^{2}\left(\left|D_{i} \phi_{1}\right|^{2}+\left|D_{i} \phi_{2}\right|^{2}\right)\right. \\
& \left.+\frac{4 \pi^{2}}{k^{2}}\left[\left|\phi_{1}\right|^{2}\left(\left|\phi_{2}\right|^{2}-c^{2}\right)^{2}+\left|\phi_{2}\right|^{2}\left(\left|\phi_{1}\right|^{2}+c^{2}\right)^{2}\right]\right\} \\
= & \frac{N(N-1)}{2}\left\{\frac{\left(\frac{k}{2 \pi} f_{12}^{(2)}\right)^{2}}{4\left|\phi_{1}\right|^{2}}+\frac{\left(\frac{k}{2 \pi} f_{12}^{(1)}\right)^{2}}{4\left|\phi_{2}\right|^{2}}+\sum_{i=1}^{2}\left(\left|D_{i} \phi_{1}\right|^{2}+\left|D_{i} \phi_{2}\right|^{2}\right)\right. \\
& \left.+\frac{4 \pi^{2}}{k^{2}}\left[\left|\phi_{1}\right|^{2}\left(\left|\phi_{2}\right|^{2}-c^{2}\right)^{2}+\left|\phi_{2}\right|^{2}\left(\left|\phi_{1}\right|^{2}+c^{2}\right)^{2}\right]\right\} \\
= & \frac{N(N-1)}{2}\left\{\left(\frac{\frac{k}{2 \pi} f_{12}^{(2)}}{2\left|\phi_{1}\right|} \pm \frac{2 \pi}{k}\left|\phi_{1}\right|\left(\left|\phi_{2}\right|^{2}-c^{2}\right)\right)^{2}+\left(\frac{k}{2 \pi} f_{12}^{(1)} \pm \frac{2 \pi}{k}\left|\phi_{2}\right|\left(\left|\phi_{1}\right|^{2}+c^{2}\right)\right)^{2}\right. \\
& +\left|D_{1} \phi_{1} \pm \mathrm{i} D_{2} \phi_{1}\right|^{2}+\left|D_{1} \phi_{2} \pm \mathrm{i} D_{2} \phi_{2}\right|^{2} \pm \mathrm{i}\left(\partial_{1}\left[\phi_{1} \overline{D_{2} \phi_{1}}\right]-\partial_{2}\left[\phi_{1} \overline{\left.D_{1} \phi_{1}\right]}\right)\right. \\
& \left. \pm \mathrm{i}\left(\partial_{1}\left[\phi_{2} \overline{D_{2} \phi_{2}}\right]-\partial_{2}\left[\phi_{2} \overline{D_{1} \phi_{2}}\right]\right) \pm c^{2}\left(f_{12}^{(2)}-f_{12}^{(1)}\right)\right\} .
\end{aligned}
$$

In this way we see that, when we can neglect the pure divergence terms, then the corresponding total energy admits the following lower bound:

$$
E=\int \mathcal{E} \mathrm{d} x \geq \pm \frac{N(N-1)}{2} c^{2} \int\left(f_{12}^{(2)}-f_{12}^{(1)}\right) \mathrm{d} x,
$$

which is attained by solutions of the following first-order self-dual equations:

$$
\begin{aligned}
& D_{1} \phi_{1} \pm \mathrm{i} D_{2} \phi_{1}=0 \\
& D_{1} \phi_{2} \pm \mathrm{i} D_{2} \phi_{2}=0 \\
& f_{12}^{(1)} \pm \frac{8 \pi^{2}}{k^{2}}\left|\phi_{2}\right|^{2}\left(\left|\phi_{1}\right|^{2}+c^{2}\right)=0 \\
& f_{12}^{(2)} \pm \frac{8 \pi^{2}}{k^{2}}\left|\phi_{1}\right|^{2}\left(\left|\phi_{2}\right|^{2}-c^{2}\right)=0
\end{aligned}
$$

which need to be supplemented by the Gauss-law constraints (2.41)-(2.42).

As a consequence, to obtain a self-dual field configurations, it suffices to solve for $\left(a_{1}^{(i)}, a_{2}^{(i)}, \phi_{i}\right)$ $(i=1,2)$ the self-dual equations (2.46)-(2.49) and then use (2.41)-(2.42) in order to obtain the remaining component $a_{0}^{(i)}(i=1,2)$. Such configurations identify a special class of solutions for the more general field equations (2.37)-(2.40).

The BPS-type equations (2.46)-(2.49) were first derived in [43], where the authors also provided a discussion and some numerical evidence about their solvability. Our goal in this paper is to establish with mathematical rigor existence and multiplicity results about solutions of (2.46) $-(2.49)$, also in terms of the corresponding energy levels. 
Actually, compared to other well known self-dual equations of BPS type governing Abelian and non-Abelian vortex configurations, we shall see that the set of equations (2.46)-(2.49) can support only planar non-topological type solutions. In fact, the more classical topological solutions (typical of the Abelian Higgs model) are not admissible here. So, in this case, no special "quantization" of energy can be identified in terms of energy minimizer over appropriate topological classes. This fact also excludes the presence of an energy gap along solutions of (2.46)-(2.49). On the contrary, we show that any (positive) energy level can be attained together with the equal sign in (2.45).

In addition, we point out that, for planar solutions of (2.46) (2.49), the usual non-topological boundary condition:

$$
\left|\phi_{i}\right|^{2} \rightarrow 0 \quad \text { as } \quad|x| \rightarrow \infty, \quad i=1,2
$$

is necessary but not sufficient to accomplish the finite-energy condition.

In this paper we shall be able to handle such a new situation in case the complex scalar fields admit the same unique zero with possible different multiplicities. Recall that, on the basis of equations (2.46) and (2.47), we know that the zeros of the complex scalars $\phi_{1}$ and $\phi_{2}$ are discrete and with integral multiplicities (see [32]). Furthermore, in a self-dual regime, such zeros do not interact, and so they can be arbitrarily prescribed. Thus, we focus to the case where both $\phi_{1}$ and $\phi_{2}$ admit the same unique zero at a point $p$ with multiplicities $N_{1}$ and $N_{2}$, respectively. This fact will allow us to look for solutions radially symmetric about such point.

Since solutions of equations (2.46) $-(2.49)$ with the lower and upper sign are related via the relation $\left(a_{1}^{(i)}, a_{2}^{(i)}, \phi_{i}\right) \leftrightarrow\left(-a_{1}^{(i)},-a_{2}^{(i)}, \overline{\phi_{i}}\right)(i=1,2)$, in what follows we only deal with the case where we specify the lower sign in (2.46) $-(2.49)$.

We show that in such case the total fluxes:

$$
\Phi_{i}=\int_{\mathbb{R}^{2}} f_{12}^{(i)} \mathrm{d} x, \quad i=1,2
$$

must satisfy:

$$
\frac{1}{2 \pi} \Phi_{2} \in\left(N_{2}+1, N_{1}+N_{2}+1\right) \quad \text { and } \quad \frac{1}{2 \pi} \Phi_{1} \in\left(N_{1}+N_{2}+1, \infty\right)
$$

However, such set of conditions are not sufficient, in the sense that we cannot assign independently the values of $\Phi_{1}$ and $\Phi_{2}$, as prescribed by (2.52).

We shall discuss below to what extent the necessary conditions (2.52) turn into sufficient ones. But first to check that $\Phi_{1}$ and $\Phi_{2}$ influence each other, we state the following result which describes a very special class of field configurations, solutions of (2.46)-(2.49), characterized by a physically relevant "concentration" property.

Theorem 2.1 Let $k>0, \sigma>0, p \in \mathbb{R}^{2}$ and $N_{1}, N_{2} \in \mathbb{N}$. There exists $\varepsilon_{0}>0$ such that $\forall \varepsilon \in\left(0, \varepsilon_{0}\right)$ the system (2.46) -(2.49) admits a field configuration: $\left(a_{1}^{(i), \varepsilon}, a_{2}^{(i), \varepsilon}, \phi_{i}^{\varepsilon}\right)(i=1,2)$ where $p$ is the only zero of $\phi_{i}^{\varepsilon}$ with multiplicity $N_{i}(i=1,2)$, and

$$
\left|\phi_{i}^{\varepsilon}\right|^{2}=\mathrm{O}\left(|x|^{-2 \beta_{i}^{\varepsilon}}\right),\left|D_{j} \phi_{i}^{\varepsilon}\right|^{2}=\mathrm{O}\left(|x|^{-2\left(\beta_{i}^{\varepsilon}+1\right)}\right), f_{12}^{(i), \varepsilon}=\mathrm{O}\left(|x|^{-2 \beta_{i}^{\varepsilon}}\right), \text { as }|x| \rightarrow \infty
$$

with $\beta_{1}^{\varepsilon}=N_{2}+1+\mathrm{o}(1)$ and $\beta_{2}^{\varepsilon}=N_{1}+1+\mathrm{o}(1)$ as $\varepsilon \rightarrow 0^{+}$. 
Furthermore, as $\varepsilon \rightarrow 0^{+}$:

$$
\begin{aligned}
& \frac{1}{2 \pi} f_{12}^{(i), \varepsilon} \rightarrow\left(N_{1}+N_{2}+1\right) \delta_{p} \quad \text { weakly in the sense of measure in } \mathbb{R}^{2}(i=1,2), \\
& f_{12}^{(1), \varepsilon}-f_{12}^{(2), \varepsilon} \rightarrow 0 \quad \text { in } \quad L^{1}\left(\mathbb{R}^{2}\right) .
\end{aligned}
$$

In particular, as $\varepsilon \rightarrow 0^{+}$:

$$
\begin{aligned}
& \frac{1}{2 \pi} \Phi_{i, \varepsilon}=\frac{1}{2 \pi} \int_{\mathbb{R}^{2}} f_{12}^{(i), \varepsilon} \mathrm{d} x \rightarrow N_{1}+N_{2}+1(i=1,2), \\
& E_{\varepsilon}=\frac{N(N-1) \sigma k}{2 \pi} \int_{\mathbb{R}^{2}}\left(f_{12}^{(1), \varepsilon}-f_{12}^{(2), \varepsilon}\right) \mathrm{d} x \rightarrow 0 .
\end{aligned}
$$

Notice that the configurations constructed in Theorem 2.1 cover only a very tiny part of full range of values specified in (2.52), however, they confirm the fact that the values of the fluxes $\Phi_{1}$ and $\Phi_{2}$ are tied to each other. This suggests that we are free to prescribe only one of the two fluxes, according to (2.52). We prove this fact in the following:

Theorem 2.2 Let $k>0$ and $\sigma>0$. For any point $p \in \mathbb{R}^{2}$ and $N_{1}, N_{2} \in \mathbb{N}$, the condition:

$$
\frac{1}{2 \pi} \Phi_{2} \in\left(N_{2}+1, N_{1}+N_{2}+1\right) \quad\left(\text { or } \quad \frac{1}{2 \pi} \Phi_{1} \in\left(N_{1}+N_{2}+1, \infty\right)\right)
$$

is necessary and sufficient for the existence of a finite-energy field configuration $\left(a_{1}^{(i)}, a_{2}^{(i)}, \phi_{i}\right)(i=$ $1,2)$ solutions of (2.46) -(2.49) in $\mathbb{R}^{2}$, with $\phi_{i}$ vanishing exactly at $p$ with multiplicities $N_{i}(i=1,2)$.

Furthermore, the following asymptotic estimates hold:

$$
\left|\phi_{i}\right|^{2}=\mathrm{O}\left(|x|^{-2 \beta_{i}}\right),\left|D_{j} \phi_{i}\right|^{2}=\mathrm{O}\left(|x|^{-2\left(\beta_{i}+1\right)}\right), f_{12}^{(i)}=\mathrm{O}\left(|x|^{-2 \beta_{i}}\right) \text {, as }|x| \rightarrow \infty
$$

with $\beta_{i} \equiv \frac{1}{2 \pi} \Phi_{i}-N_{i}(i=1,2)$ satisfying: $\beta_{1} \in\left(N_{2}+1, \infty\right)$ and $\beta_{2} \in\left(1, N_{1}+1\right)$, and more specifically,

$$
\begin{aligned}
& 0<\left(\beta_{1}-1\right)\left(\beta_{2}-1\right)<\left(N_{1}+1\right)\left(N_{2}+1\right), \\
& \beta_{2}\left(\beta_{1}-1\right)>N_{2}\left(N_{1}+1\right) \\
& 0<\beta_{1}\left(\beta_{2}-1\right)<N_{1}\left(N_{2}+1\right) .
\end{aligned}
$$

Theorem 2.1 and Theorem 2.2 also indicate that the set of admissible fluxes $\left(\Phi_{1}, \Phi_{2}\right)$ describes a region in the plane bounded by two curves parameterized by $\Phi_{1}$ (or $\Phi_{2}$ ), emanating form a zero-energy configuration.

The following multiplicity result substantiates this fact.

Theorem 2.3 Let $k>0, \sigma>0, p \in \mathbb{R}^{2}$ and $N_{1}, N_{2} \in \mathbb{N}$. For any $\gamma_{2} \in\left(N_{2}+1, N_{1}+N_{2}+1\right)$ (or $\left.\gamma_{1}>N_{1}+1\right)$, there exits a 1-parameter family of finite-energy solutions of (2.46)-(2.49) in $\mathbb{R}^{2}$ such that the complex scalar fields $\phi_{i}$ vanish only at $p$ with multiplicity $N_{1}$ and $N_{2}$ respectively, and

$$
\gamma_{2}=\frac{1}{2 \pi} \Phi_{2} \quad\left(\text { or } \gamma_{1}=\frac{1}{2 \pi} \Phi_{1}\right) .
$$


Equivalently, for any assigned energy level $E>0$, there exists a 1-parameter family of solutions as described above, which all carry total energy E, namely:

$$
E=\frac{N(N-1) \sigma k}{4 \pi} \int_{\mathbb{R}^{2}}\left(f_{12}^{(1)}-f_{12}^{(2)}\right) \mathrm{d} x .
$$

For all such solutions, the asymptotic estimates (2.59) hold together with (2.60)-(2.62).

To establish the above results, we reduce (2.46) $-(2.49)$ into a $2 \times 2$ system of second-order elliptic equations. To this purpose, let the zeros of $\phi_{i}$ have multiplicity $N_{i} \in \mathbb{N}(i=1,2)$ and be given as follows:

$$
p_{i 1,}, \cdots, p_{i N_{i}}, \quad i=1,2,
$$

each repeated according to its multiplicity.

Set

$$
u=\ln \left|\phi_{1}\right|^{2}-\ln \frac{\sigma k}{2 \pi}, \quad v=\ln \left|\phi_{2}\right|^{2}-\ln \frac{\sigma k}{2 \pi} .
$$

By means of the relations (see e.g. [63]):

$$
\begin{aligned}
& \phi_{1}=\sqrt{\frac{\sigma k}{2 \pi}} \exp \left(\frac{1}{2} u(x)+\mathrm{i} \sum_{s=1}^{N_{1}} \arg \left(x-p_{1 s}\right)\right), \\
& \phi_{2}=\sqrt{\frac{\sigma k}{2 \pi}} \exp \left(\frac{1}{2} v(x)+\mathrm{i} \sum_{s=1}^{N_{2}} \arg \left(x-p_{2 s}\right)\right), \\
& a_{1}^{(1)}(x)=-\operatorname{Re}\left\{\mathrm{i} \bar{\partial} \ln \phi_{1}(x)\right\}, \quad a_{2}^{(1)}(x)=-\operatorname{Im}\left\{\mathrm{i} \bar{\partial} \ln \phi_{1}(x)\right\}, \\
& a_{1}^{(2)}(x)=-\operatorname{Re}\left\{\mathrm{i} \bar{\partial} \ln \phi_{2}(x)\right\}, \quad a_{2}^{(2)}(x)=-\operatorname{Im}\left\{\mathrm{i} \bar{\partial} \ln \phi_{2}(x)\right\}, \\
& \left|\phi_{1}\right|^{2}=\frac{\sigma k}{2 \pi} \mathrm{e}^{u}, \quad\left|\phi_{2}\right|^{2}=\frac{\sigma k}{2 \pi} \mathrm{e}^{v}, \\
& \left|D_{j} \phi_{1}\right|^{2}=\frac{\sigma k}{4 \pi} \mathrm{e}^{u}|\nabla u|^{2}, \quad\left|D_{j} \phi_{2}\right|^{2}=\frac{\sigma k}{4 \pi} \mathrm{e}^{v}|\nabla v|^{2},
\end{aligned}
$$

with $\bar{\partial}=\frac{1}{2}\left(\partial_{1}+\mathrm{i} \partial_{2}\right)$, it is standard to see that,

$$
-\Delta u=2 f_{12}^{(1)}-4 \pi \sum_{s=1}^{N_{1}} \delta_{p_{1 s}} \quad \text { and } \quad-\Delta v=2 f_{12}^{(2)}-4 \pi \sum_{s=1}^{N_{2}} \delta_{p_{2 s}},
$$

where $\delta_{p}$ denotes the Dirac measure concentrated at $p \in \mathbb{R}^{2}$.

Therefore, we find that, problem (2.46)-(2.49) can be equivalently reformulated in terms of the unknown $(u, v)$ satisfying the following system of nonlinear elliptic equations:

$$
\left\{\begin{array}{l}
-\Delta u=\lambda \mathrm{e}^{v}\left(\mathrm{e}^{u}+1\right)-4 \pi \sum_{s=1}^{N_{1}} \delta_{p_{1 s}} \text { in } \mathbb{R}^{2}, \\
-\Delta v=\lambda \mathrm{e}^{u}\left(\mathrm{e}^{v}-1\right)-4 \pi \sum_{s=1}^{N_{2}} \delta_{p_{2 s}} \text { in } \mathbb{R}^{2},
\end{array}\right.
$$


with

$$
\lambda \equiv 4 \sigma^{2}>0 .
$$

In other words, any solution of (2.46) -(2.49) with the zeros of $\phi_{i}$ given by (2.65) can be expressed in terms of (2.67)-(2.70) with $(u, v)$ satisfying (2.74). Viceversa, from every solution of (2.74) we can recover a field configuration, $\left(a_{1}^{(i)}, a_{2}^{(i)}, \phi_{i}\right)(\mathrm{i}=1,2)$, solution of (2.46) $-(2.49)$, via the relations (2.67) -(2.70), such that the zeros of $\phi_{i}(i=1,2)$ are given exactly by (2.65).

In view of (2.48), (2.49), (2.51) and (2.67)-(2.70), to obtain a planar self-dual field configuration which carries finite total energy and fluxes, we need to solve (2.74) over $\mathbb{R}^{2}$ under the integrability conditions:

$$
\mathrm{e}^{u}, \mathrm{e}^{v}, \mathrm{e}^{u+v} \in L^{1}\left(\mathbb{R}^{2}\right) .
$$

In particular, (2.76) implies that we must satisfy the non-topological boundary conditions:

$$
u \rightarrow-\infty, \quad v \rightarrow-\infty \quad \text { as } \quad|x| \rightarrow \infty
$$

However, we anticipate that, contrary to other self-dual equations, for solutions of (2.74) the boundary conditions (2.77) do not always imply (2.76). Furthermore, under the standard transformation (natural for Liouville systems):

$$
u(x) \rightarrow u\left(\frac{x}{\varepsilon}\right)+2 \ln \frac{1}{\varepsilon}, \quad v(x) \rightarrow v\left(\frac{x}{\varepsilon}\right)+2 \ln \frac{1}{\varepsilon},
$$

with $\varepsilon>0$ small, problem (2.74) transforms into a "perturbation" of an indefinite Liouville system, which admits no solutions under the integrability condition (2.76). In other words, under (2.76), for the given system we have no hope to find solutions "bifurcating" from Liouville solutions.

On the contrary, by changing the point of view, we find that, it is the "interacting" term $\mathrm{e}^{u+v}$ to play the role of "leading" term within a (more appropriate) perturbation approach. So, under a suitable scaling, it will be the term $\mathrm{e}^{u+v}$ to behave as a Liouville solution. Interestingly, such an understanding will help us also to find solutions away from the perturbation regime.

To avoid additional technicalities in carrying out such program, we will focus to the case where all the zeros of the complex scalars are superimposed at a point, which (after a translation), we may take to be the origin. Namely, we set

$$
p_{i 1}=\cdots=p_{i N_{i}}=0, \quad i=1,2 .
$$

Moreover, by rescaling:

$$
u(x) \rightarrow u\left(\frac{x}{\sqrt{\lambda}}\right) \text { and } v(x) \rightarrow v\left(\frac{x}{\sqrt{\lambda}}\right),
$$

we can further assume that $\lambda=1$. So, we need to study the solvability of the system:

$$
\left\{\begin{array}{l}
-\Delta u=\mathrm{e}^{v}\left(\mathrm{e}^{u}+1\right)-4 \pi N_{1} \delta_{0} \quad \text { in } \mathbb{R}^{2}, \\
-\Delta v=\mathrm{e}^{u}\left(\mathrm{e}^{v}-1\right)-4 \pi N_{2} \delta_{0} \text { in } \mathbb{R}^{2},
\end{array}\right.
$$

with $N_{1}, N_{2} \in \mathbb{N}$, under the integrability conditions specified in (2.76).

By analyzing entire radial solutions of (2.80) about the origin, we check that, even when they realized the boundary condition (2.77), they may fail to satisfy (2.76). Therefore, our main task will be to identify suitable set of initial conditions which yield to (radial) solutions of (2.80) satisfying (2.76), see Theorem 6.1 for details. More precisely, we shall analyze their multiplicity along any assigned energy level or at fixed fluxes. 


\section{Necessary conditions for solvability}

In this section we derive necessary conditions for the solvability of the problem:

$$
\left\{\begin{array}{l}
-\Delta u=\mathrm{e}^{v}\left(\mathrm{e}^{u}+1\right)-4 \pi N_{1} \delta_{0} \quad \text { in } \quad \mathbb{R}^{2}, \\
-\Delta v=\mathrm{e}^{u}\left(\mathrm{e}^{v}-1\right)-4 \pi N_{2} \delta_{0} \quad \text { in } \quad \mathbb{R}^{2}, \\
\mathrm{e}^{u}, \mathrm{e}^{v}, \mathrm{e}^{u+v} \in L^{1}\left(\mathbb{R}^{2}\right) .
\end{array}\right.
$$

To this purpose we recall that, by an approach introduced by Chen-Li [13, 14] for Liouville equations (based on potential estimates), it is possible to show that every solution $(u, v)$ of (3.1) must admit the following asymptotic behavior at $\infty$ :

$$
\left\{\begin{array}{l}
u(x)=-\left(\frac{1}{2 \pi} \int_{\mathbb{R}^{2}} \mathrm{e}^{v}\left(\mathrm{e}^{u}+1\right) \mathrm{d} x-2 N_{1}\right) \ln |x|+\mathrm{O}(1) \quad \text { as } \quad|x| \rightarrow \infty \\
v(x)=-\left(\frac{1}{2 \pi} \int_{\mathbb{R}^{2}} \mathrm{e}^{u}\left(\mathrm{e}^{v}-1\right) \mathrm{d} x-2 N_{2}\right) \ln |x|+\mathrm{O}(1) \quad \text { as } \quad|x| \rightarrow \infty \\
r \partial_{r} u \rightarrow 2 N_{1}-\frac{1}{2 \pi} \int_{\mathbb{R}^{2}} \mathrm{e}^{v}\left(\mathrm{e}^{u}+1\right) \mathrm{d} x, \quad \partial_{\theta} u \rightarrow 0 \quad \text { as } \quad|x| \rightarrow \infty \\
r \partial_{r} v \rightarrow 2 N_{2}-\frac{1}{2 \pi} \int_{\mathbb{R}^{2}} \mathrm{e}^{u}\left(\mathrm{e}^{v}-1\right) \mathrm{d} x, \quad \partial_{\theta} v \rightarrow 0 \quad \text { as } \quad|x| \rightarrow \infty
\end{array}\right.
$$

with $(r, \theta)$ the polar coordinates in $\mathbb{R}^{2}$. More precisely, to establish (3.2), we can argue exactly as for the proof of Theorem 1.1 in [10] by using Lemma 1.1 therein.

Therefore, by the integrability conditions, and by recalling (2.73), we find that,

$$
\left\{\begin{array}{l}
\frac{1}{\pi} \Phi_{1}=\frac{1}{2 \pi} \int_{\mathbb{R}^{2}} \mathrm{e}^{v}\left(\mathrm{e}^{u}+1\right) \mathrm{d} x>2\left(N_{1}+1\right), \\
\frac{1}{\pi} \Phi_{2}=\frac{1}{2 \pi} \int_{\mathbb{R}^{2}} \mathrm{e}^{u}\left(\mathrm{e}^{v}-1\right) \mathrm{d} x>2\left(N_{2}+1\right) .
\end{array}\right.
$$

Furthermore, we can use the above decay estimates at infinity to derive the following Pohozaev identity:

Lemma 3.1 Every solution $(u, v)$ of (3.1) satisfies:

$$
\begin{aligned}
& \left(\frac{1}{2 \pi} \int_{\mathbb{R}^{2}} \mathrm{e}^{v}\left(\mathrm{e}^{u}+1\right) \mathrm{d} x-2\left(N_{1}+1\right)\right)\left(\frac{1}{2 \pi} \int_{\mathbb{R}^{2}} \mathrm{e}^{u}\left(\mathrm{e}^{v}-1\right) \mathrm{d} x-2\left(N_{2}+1\right)\right)+\frac{1}{\pi} \int_{\mathbb{R}^{2}} \mathrm{e}^{u+v} \mathrm{~d} x \\
& =4\left(N_{1}+1\right)\left(N_{2}+1\right) .
\end{aligned}
$$

Proof. By recalling the identity:

$$
-[(x \cdot \nabla v) \Delta u+(x \cdot \nabla u) \Delta v]=\operatorname{div}[x(\nabla u \cdot \nabla v)]-\operatorname{div}[(x \cdot \nabla v) \nabla u+(x \cdot \nabla u) \nabla v],
$$

and by multiplying the first equation of (3.1) by $x \cdot \nabla v$, and the second equation of (3.1) by $x \cdot \nabla u$, we can use integration by parts over $\Omega \equiv\left\{x \in \mathbb{R}^{2}|\delta<| x \mid<R\right\}$ for $0<\delta<R$ and obtain:

$$
\begin{array}{r}
\int_{\partial \Omega}(\nabla u \cdot \nabla v) x \cdot \nu \mathrm{d} S-\int_{\partial \Omega}[(x \cdot \nabla v) \nabla u \cdot \nu+(x \cdot \nabla u) \nabla v \cdot \nu] \mathrm{d} S \\
=\int_{\partial \Omega}\left[\mathrm{e}^{v}\left(\mathrm{e}^{u}+1\right)+\mathrm{e}^{u}\left(\mathrm{e}^{v}-1\right)\right] x \cdot \nu \mathrm{d} S-\int_{\partial \Omega} \mathrm{e}^{u+v} x \cdot \nu \mathrm{d} S-2 \int_{\Omega}\left(\mathrm{e}^{u+v}+\mathrm{e}^{v}-\mathrm{e}^{u}\right) \mathrm{d} x .
\end{array}
$$


Since after integration by parts, we also find that:

$$
\begin{gathered}
-\int_{\partial \Omega} \nabla u \cdot \nu \mathrm{d} S=\int_{\Omega} \mathrm{e}^{v}\left(\mathrm{e}^{u}+1\right) \mathrm{d} x, \\
-\int_{\partial \Omega} \nabla v \cdot \nu \mathrm{d} S=\int_{\Omega} \mathrm{e}^{u}\left(\mathrm{e}^{v}-1\right) \mathrm{d} x,
\end{gathered}
$$

by combining the identities above, we obtain:

$$
\begin{array}{r}
-\int_{\partial \Omega}[2(\nabla u \cdot \nu+\nabla v \cdot \nu)-(\nabla u \cdot \nabla v) x \cdot \nu+(x \cdot \nabla v) \nabla u \cdot \nu+(x \cdot \nabla u) \nabla v \cdot \nu] \mathrm{d} S \\
=\int_{\partial \Omega}\left[\mathrm{e}^{v}\left(\mathrm{e}^{u}+1\right)+\mathrm{e}^{u}\left(\mathrm{e}^{v}-1\right)\right] x \cdot \nu \mathrm{d} S-\int_{\partial \Omega} \mathrm{e}^{u+v} x \cdot \nu \mathrm{d} S+2 \int_{\Omega} \mathrm{e}^{u+v} \mathrm{~d} x .
\end{array}
$$

Therefore, we can use the asymptotic estimates (3.2), and the logarithmal behavior of $u, v$ at the origin, to see that, by letting $\delta \rightarrow 0$ and $R \rightarrow \infty$ in (3.9), we can obtain the desired identity (3.4).

At this point, by straightforward calculation, we can rewrite (3.4) in the following equivalent forms:

$$
\begin{aligned}
& \left(\frac{1}{2 \pi} \int_{\mathbb{R}^{2}} \mathrm{e}^{u}\left(\mathrm{e}^{v}-1\right) \mathrm{d} x-2\left(N_{1}+N_{2}+1\right)\right) \frac{1}{2 \pi} \int_{\mathbb{R}^{2}} \mathrm{e}^{u+v} \mathrm{~d} x+\frac{N_{1}+1}{\pi} \int_{\mathbb{R}^{2}} \mathrm{e}^{u} \mathrm{~d} x \\
& +\left(\frac{1}{2 \pi} \int_{\mathbb{R}^{2}} \mathrm{e}^{u}\left(\mathrm{e}^{v}-1\right) \mathrm{d} x-2\left(N_{2}+1\right)\right) \frac{1}{2 \pi} \int_{\mathbb{R}^{2}} \mathrm{e}^{v} \mathrm{~d} x=0
\end{aligned}
$$

and

$$
\begin{aligned}
& \left(\frac{1}{2 \pi} \int_{\mathbb{R}^{2}} \mathrm{e}^{v}\left(\mathrm{e}^{u}+1\right) \mathrm{d} x-2\left(N_{1}+N_{2}+1\right)\right) \frac{1}{2 \pi} \int_{\mathbb{R}^{2}} \mathrm{e}^{u+v} \mathrm{~d} x-\frac{N_{2}+1}{\pi} \int_{\mathbb{R}^{2}} \mathrm{e}^{v} \mathrm{~d} x \\
& -\left(\frac{1}{2 \pi} \int_{\mathbb{R}^{2}} \mathrm{e}^{v}\left(\mathrm{e}^{u}+1\right) \mathrm{d} x-2\left(N_{1}+1\right)\right) \frac{1}{2 \pi} \int_{\mathbb{R}^{2}} \mathrm{e}^{u} \mathrm{~d} x=0 .
\end{aligned}
$$

Consequently, in view of (3.3), we derive that necessarily there must hold:

$$
2\left(N_{2}+1\right)<\frac{1}{\pi} \Phi_{2}=\frac{1}{2 \pi} \int_{\mathbb{R}^{2}} \mathrm{e}^{u}\left(\mathrm{e}^{v}-1\right) \mathrm{d} x<2\left(N_{1}+N_{2}+1\right)<\frac{1}{2 \pi} \int_{\mathbb{R}^{2}} \mathrm{e}^{v}\left(\mathrm{e}^{u}+1\right) \mathrm{d} x=\frac{1}{\pi} \Phi_{1}
$$

which express the necessary conditions claimed in (2.52) for the solvability of (3.1).

Our next goal in the following sections is to show that, each of the condition above, concerning either $\Phi_{1}$ or $\Phi_{2}$ is also sufficient for the solvability of (3.1).

\section{A perturbation approach and proof of Theorem 2.1}

In this section we establish an existence theorem for the system (3.1) by means of a perturbation approach, from which we can prove Theorem 2.1.

For any $\varepsilon>0$, we consider the scaled functions:

$$
u_{\varepsilon}(x)=u(\varepsilon x)+\ln \varepsilon, \quad v_{\varepsilon}(x)=v(\varepsilon x)+\ln \varepsilon
$$


satisfying:

$$
\left\{\begin{array}{l}
-\Delta u_{\varepsilon}=\mathrm{e}^{u_{\varepsilon}+v_{\varepsilon}}+\varepsilon \mathrm{e}^{v_{\varepsilon}}-4 \pi N_{1} \delta_{0} \quad \text { in } \mathbb{R}^{2}, \\
-\Delta v_{\varepsilon}=\mathrm{e}^{u_{\varepsilon}+v_{\varepsilon}}-\varepsilon \mathrm{e}^{u_{\varepsilon}}-4 \pi N_{2} \delta_{0} \text { in } \mathbb{R}^{2}, \\
\mathrm{e}^{u_{\varepsilon}}, \mathrm{e}^{v_{\varepsilon}}, \mathrm{e}^{u_{\varepsilon}+v_{\varepsilon}} \in L^{1}\left(\mathbb{R}^{2}\right)
\end{array}\right.
$$

and

$$
\int_{\mathbb{R}^{2}} \mathrm{e}^{u_{\varepsilon}+v_{\varepsilon}} \mathrm{d} x=\int_{\mathbb{R}^{2}} \mathrm{e}^{u+v} \mathrm{~d} x, \int_{\mathbb{R}^{2}} \mathrm{e}^{u_{\varepsilon}} \mathrm{d} x=\varepsilon \int_{\mathbb{R}^{2}} \mathrm{e}^{u} \mathrm{~d} x, \int_{\mathbb{R}^{2}} \mathrm{e}^{v_{\varepsilon}} \mathrm{d} x=\varepsilon \int_{\mathbb{R}^{2}} \mathrm{e}^{v} \mathrm{~d} x
$$

Formally, by letting $\varepsilon \rightarrow 0^{+}$, (4.2) can be viewed as a "perturbation" of the following problem:

$$
\left\{\begin{array}{l}
-\Delta u_{0}=\mathrm{e}^{u_{0}+v_{0}}-4 \pi N_{1} \delta_{0} \quad \text { in } \quad \mathbb{R}^{2}, \\
-\Delta v_{0}=\mathrm{e}^{u_{0}+v_{0}}-4 \pi N_{2} \delta_{0} \quad \text { in } \quad \mathbb{R}^{2} \\
\mathrm{e}^{u_{0}}, \mathrm{e}^{v_{0}}, \mathrm{e}^{u_{0}+v_{0}} \in L^{1}\left(\mathbb{R}^{2}\right) .
\end{array}\right.
$$

To classify solutions of the system (4.4), simply we rewrite it equivalently as follows:

$$
\left\{\begin{array}{l}
-\Delta\left(u_{0}+v_{0}\right)=2 \mathrm{e}^{u_{0}+v_{0}}-4 \pi\left(N_{1}+N_{2}\right) \delta_{0} \quad \text { in } \mathbb{R}^{2}, \\
-\Delta\left(u_{0}-v_{0}\right)=-4 \pi\left(N_{1}-N_{2}\right) \delta_{0} \quad \text { in } \quad \mathbb{R}^{2}, \\
\mathrm{e}^{u_{0}}, \mathrm{e}^{v_{0}}, \mathrm{e}^{u_{0}+v_{0}} \in L^{1}\left(\mathbb{R}^{2}\right),
\end{array}\right.
$$

and so, by the result of [55] about Liouville equation, we can explicitly express solutions of (4.5) in terms of the free parameters $a \in \mathbb{C}, \lambda>0$ and $c \in \mathbb{R}$, and (in complex notations) as follows:

$$
\left\{\begin{array}{l}
\left(u_{0}+v_{0}\right)(z)=\ln \frac{4\left(N_{1}+N_{2}+1\right)^{2} \lambda|z|^{2\left(N_{1}+N_{2}\right)}}{\left(1+\lambda\left|z^{N_{1}+N_{2}+1}+a\right|^{2}\right)^{2}} \\
\left(u_{0}-v_{0}\right)(z)=\left(N_{1}-N_{2}\right) \ln |z|^{2}+c .
\end{array}\right.
$$

We focus around the following (normalized) solution of (4.4):

$$
\left\{\begin{array}{l}
u_{0}=\ln \frac{2\left(N_{1}+N_{2}+1\right)|z|^{2 N_{1}}}{\left(1+|z|^{2\left(N_{1}+N_{2}+1\right)}\right)} \\
v_{0}=\ln \frac{2\left(N_{1}+N_{2}+1\right)|z|^{2 N_{2}}}{\left(1+|z|^{2\left(N_{1}+N_{2}+1\right)}\right)}
\end{array}\right.
$$

and set,

$$
\rho(r) \equiv 2 \mathrm{e}^{u_{0}+v_{0}}=\frac{8\left(N_{1}+N_{2}+1\right)^{2} r^{2\left(N_{1}+N_{2}\right)}}{\left(1+r^{2\left(N_{1}+N_{2}+1\right)}\right)^{2}}, \quad r=|z| .
$$

We aim to seek solutions of (4.2) in the form:

$$
u_{\varepsilon}(r)=u_{0}(r)+\varepsilon u_{1}(r), \quad v_{\varepsilon}(r)=v_{0}(r)+\varepsilon v_{1}(r),
$$

where $\left(u_{1}, v_{1}\right)$ satisfies:

$$
\left\{\begin{array}{l}
-\Delta u_{1}=\mathrm{e}^{u_{0}+v_{0}}\left(\frac{\mathrm{e}^{\varepsilon\left(u_{1}+v_{1}\right)}-1}{\varepsilon}\right)+\mathrm{e}^{v_{0}+\varepsilon v_{1}}, \\
-\Delta v_{1}=\mathrm{e}^{u_{0}+v_{0}}\left(\frac{\mathrm{e}^{\varepsilon\left(u_{1}+v_{1}\right)}-1}{\varepsilon}\right)-\mathrm{e}^{u_{0}+\varepsilon u_{1}}
\end{array}\right.
$$


Formally, by setting $\varepsilon \rightarrow 0^{+}$, we see that (4.9) yields to the following system:

$$
\left\{\begin{array}{l}
-\Delta u_{2}=\mathrm{e}^{u_{0}+v_{0}}\left(u_{2}+v_{2}\right)+\mathrm{e}^{v_{0}}, \\
-\Delta v_{2}=\mathrm{e}^{u_{0}+v_{0}}\left(u_{2}+v_{2}\right)-\mathrm{e}^{u_{0}},
\end{array}\right.
$$

or equivalently:

$$
\left\{\begin{array}{l}
-\Delta\left(u_{2}+v_{2}\right)=2 \mathrm{e}^{u_{0}+v_{0}}\left(u_{2}+v_{2}\right)-\mathrm{e}^{u_{0}}+\mathrm{e}^{v_{0}}, \\
-\Delta\left(u_{2}-v_{2}\right)=\mathrm{e}^{u_{0}}+\mathrm{e}^{v_{0}},
\end{array}\right.
$$

with $u_{0}$ and $v_{0}$ specified in (4.6).

We are going to exhibit in the following an explicit radial solution $\left(u_{2}(r), v_{2}(r)\right)$ of (4.11), see (4.35)-(4.36) below.

Therefore, we use the decomposition:

$$
u_{1}(r)=u_{2}(r)+u_{3}(r), \quad v_{1}(r)=v_{2}(r)+v_{3}(r)
$$

with $u_{3}(r)$ and $v_{3}(r)$ the error terms, going to zero in suitable norm as $\varepsilon \rightarrow 0^{+}$. So via (4.8), (4.12) we determine a solution of the original problem (4.9), as soon as we solve:

$$
\left\{\begin{array}{l}
\Delta u_{3}+\mathrm{e}^{u_{0}+v_{0}}\left(\frac{\mathrm{e}^{\varepsilon\left(u_{2}+v_{2}+u_{3}+v_{3}\right)}-1}{\varepsilon}-\left(u_{2}+v_{2}\right)\right)+\mathrm{e}^{v_{0}}\left(\mathrm{e}^{\varepsilon\left(v_{2}+v_{3}\right)}-1\right)=0, \\
\Delta v_{3}+\mathrm{e}^{u_{0}+v_{0}}\left(\frac{\mathrm{e}^{\varepsilon\left(u_{2}+v_{2}+u_{3}+v_{3}\right)}-1}{\varepsilon}-\left(u_{2}+v_{2}\right)\right)-\mathrm{e}^{u_{0}}\left(\mathrm{e}^{\varepsilon\left(u_{2}+u_{3}\right)}-1\right)=0,
\end{array}\right.
$$

with the required integrability conditions.

Set

$$
\left\{\begin{aligned}
P_{1}\left(u_{3}, v_{3}, \varepsilon\right) \equiv & \Delta u_{3}+\mathrm{e}^{u_{0}+v_{0}}\left(\frac{\mathrm{e}^{\varepsilon\left(u_{2}+v_{2}+u_{3}+v_{3}\right)}-1}{\varepsilon}-\left(u_{2}+v_{2}\right)\right) \\
& +\mathrm{e}^{v_{0}}\left(\mathrm{e}^{\varepsilon\left(v_{2}+v_{3}\right)}-1\right), \\
P_{2}\left(u_{3}, v_{3}, \varepsilon\right) \equiv & \Delta v_{3}+\mathrm{e}^{u_{0}+v_{0}}\left(\frac{\mathrm{e}^{\varepsilon\left(u_{2}+v_{2}+u_{3}+v_{3}\right)}-1}{\varepsilon}-\left(u_{2}+v_{2}\right)\right) \\
& -\mathrm{e}^{u_{0}}\left(\mathrm{e}^{\varepsilon\left(u_{2}+u_{3}\right)}-1\right),
\end{aligned}\right.
$$

and

$$
P_{i}(0,0,0) \equiv 0, \quad i=1,2 .
$$

Following [9], it is natural to consider the operator $P_{i}$ to act between the following spaces:

$$
\begin{aligned}
& Y_{\alpha} \equiv\left\{w \in W_{l o c}^{2,2}\left(\mathbb{R}^{2}\right): \quad \Delta w \in X_{\alpha}, \quad \frac{w}{\left(1+|z|^{1+\frac{\alpha}{2}}\right)} \in L^{2}\left(\mathbb{R}^{2}\right)\right\}, \quad 0<\alpha<1, \\
& X_{\alpha} \equiv\left\{w \in L_{l o c}^{2}\left(\mathbb{R}^{2}\right): \quad\left(1+|z|^{2+\alpha}\right) w^{2} \in L^{1}\left(\mathbb{R}^{2}\right)\right\}, \quad 0<\alpha<1 .
\end{aligned}
$$

Clearly, $Y_{\alpha}$ and $X_{\alpha}$ define Hilbert spaces equipped respectively with the following scalar products:

$$
\begin{aligned}
(u, v)_{Y_{\alpha}} & \equiv(\Delta u, \Delta v)_{X_{\alpha}}+\int_{\mathbb{R}^{2}} \frac{u v}{\left(1+|z|^{2+\alpha}\right)} \mathrm{d} z \\
(u, v)_{X_{\alpha}} & \equiv \int_{\mathbb{R}^{2}}\left(1+|z|^{2+\alpha}\right) u v \mathrm{~d} z
\end{aligned}
$$


and induced norms denoted respectively by $\|\cdot\|_{Y_{\alpha}}$ and $\|\cdot\|_{X_{\alpha}}$.

Moreover, we let $Y_{\alpha}^{r}$ and $X_{\alpha}^{r}$ respectively to be the subspaces of $Y_{\alpha}$ and $X_{\alpha}$ consisting of radial functions.

We recall the following properties of functions in $Y_{\alpha}$, whose proof can be found in [60].

Lemma 4.1 ([60]) Let $\alpha \in(0,1), w \in Y_{\alpha}$.

(i) If $w \in Y_{\alpha}$ is harmonic, then $w$ is a constant.

(ii) The following estimates hold, $\forall w \in Y_{\alpha}$ :

$$
\begin{aligned}
& |w(z)| \leq C\|w\|_{Y_{\alpha}} \ln (1+|z|), \forall z \in \mathbb{R}^{2}, \\
& \|\nabla w\|_{L^{p}} \leq C_{p}\|w\|_{Y_{\alpha}}, \quad \forall p>2
\end{aligned}
$$

with $C$ and $C_{p}$ positive constants depending only on $\alpha$ and $(\alpha, p)$, respectively.

It is easy to check that the operator:

$$
P: Y_{\alpha}^{r} \times Y_{\alpha}^{r} \times \mathbb{R} \rightarrow X_{\alpha}^{r} \times X_{\alpha}^{r},
$$

with

$$
P\left(u_{3}, v_{3}, \varepsilon\right) \equiv\left(P_{1}\left(u_{3}, v_{3}, \varepsilon\right), P_{2}\left(u_{3}, v_{3}, \varepsilon\right)\right)
$$

is well defined, and it is continuous and differentiable in $(0,0,0)$. Furthermore, we can compute the linearized operator $A$ at $(0,0,0)$, namely:

$$
A \equiv \frac{\partial P}{\partial\left(u_{3}, v_{3}\right)}(0,0,0): \quad Y_{\alpha}^{r} \times Y_{\alpha}^{r} \rightarrow X_{\alpha}^{r} \times X_{\alpha}^{r}
$$

and show that it takes the following form:

$$
A\left(\begin{array}{l}
\phi \\
\psi
\end{array}\right)=\left(\begin{array}{c}
\Delta \phi+\mathrm{e}^{u_{0}+v_{0}}(\phi+\psi) \\
\Delta \psi+\mathrm{e}^{u_{0}+v_{0}}(\phi+\psi)
\end{array}\right), \quad \forall \phi, \psi \in Y_{\alpha}^{r} .
$$

To identify the kernel of the operator $A$, we just need to solve the problem:

$$
\left\{\begin{array}{l}
\Delta(\phi+\psi)+\rho(\phi+\psi)=0 \\
\Delta(\phi-\psi)=0 \\
\phi, \psi \in Y_{\alpha}^{r}
\end{array}\right.
$$

with $\rho$ defined by (4.7).

To this purpose, we use Lemma 3.4.20 and Corollary 3.4.21 in [60], for the operator $L^{r}: Y_{\alpha}^{r} \rightarrow$ $X_{\alpha}^{r}$ defined as follows:

$$
L^{r} w \equiv \frac{\mathrm{d}^{2}}{\mathrm{~d} r^{2}} w+\frac{1}{r} w+\rho w
$$

More precisely, for

$$
\phi_{0}(r) \equiv \frac{1-r^{2\left(N_{1}+N_{2}+1\right)}}{1+r^{2\left(N_{1}+N_{2}+1\right)}}
$$

we have: 
Lemma 4.2 ([60]) Let $\alpha \in(0,1)$ and $N_{1}, N_{2}>0$. We have

(i) $w \in Y_{\alpha}^{r}$ satisfies $L^{r} w=0$ if and only if $w \in \operatorname{span}\left\{\phi_{0}\right\}$.

(ii) $L^{r}: Y_{\alpha}^{r} \rightarrow X_{\alpha}^{r}$ is onto. More precisely, for $f \in X_{\alpha}^{r}$, let

$$
\begin{aligned}
w(r)= & \left(\phi_{0}(r) \ln r+\frac{2}{\left(N_{1}+N_{2}+1\right)\left(1+r^{2\left(N_{1}+N_{2}+1\right)}\right)}\right) \int_{0}^{r} \phi_{0}(s) f(s) s \mathrm{~d} s \\
& -\phi_{0}(r) \int_{0}^{r}\left(\phi_{0}(s) \ln s+\frac{2}{\left(N_{1}+N_{2}+1\right)\left(1+s^{2\left(N_{1}+N_{2}+1\right)}\right)}\right) f(s) s \mathrm{~d} s,
\end{aligned}
$$

then $w \in Y_{\alpha}^{r}$ and $L^{r} w=f$. Moreover,

$$
w(r)=-c_{f} \ln r+\mathrm{O}(1), \quad w^{\prime}(r)=-\frac{c_{f}}{r}+\mathrm{O}(1), \quad \text { as } \quad r \rightarrow \infty,
$$

with

$$
c_{f} \equiv \int_{0}^{\infty} \phi_{0}(t) f(t) t \mathrm{~d} t
$$

As a consequence, from (4.26) and part $(i)$ of Lemma 4.1 and Lemma 4.2, we derive:

\section{Corollary 4.1}

$$
\operatorname{Ker} A=\left\{(w+c, w-c) \mid \quad w \in \operatorname{span}\left\{\phi_{0}\right\}, c \in \mathbb{R}\right\}
$$

Furthermore,

Lemma 4.3 The linearized operator $A: Y_{\alpha}^{r} \times Y_{\alpha}^{r} \rightarrow X_{\alpha}^{r} \times X_{\alpha}^{r}$ is onto.

Proof. For any $\left(f_{1}, f_{2}\right) \in X_{\alpha}^{r} \times X_{\alpha}^{r}$, we need to show that the system:

$$
\left\{\begin{array}{c}
\Delta \phi+\mathrm{e}^{u_{0}+v_{0}}(\phi+\psi)=f_{1}, \\
\Delta \psi+\mathrm{e}^{u_{0}+v_{0}}(\phi+\psi)=f_{2}
\end{array}\right.
$$

admits a solution in $Y_{\alpha}^{r} \times Y_{\alpha}^{r}$.

To this purpose, we write system (4.33) equivalently as follows:

$$
\left\{\begin{array}{l}
\Delta(\phi+\psi)+\rho(\phi+\psi)=f_{1}+f_{2}, \\
\Delta(\phi-\psi)=f_{1}-f_{2} .
\end{array}\right.
$$

So, by Lemma 4.2, we can solve the first equation of (4.34) in $Y_{\alpha}^{r}$, and obtain that,

$$
\begin{aligned}
& (\phi+\psi)(r) \\
& =\left(\phi_{0}(r) \ln r+\frac{2}{\left(N_{1}+N_{2}+1\right)\left(1+r^{2\left(N_{1}+N_{2}+1\right)}\right)}\right) \int_{0}^{r} \phi_{0}(s)\left(f_{1}(s)+f_{2}(s)\right) s \mathrm{~d} s \\
& -\phi_{0}(r) \int_{0}^{r}\left(\phi_{0}(s) \ln s+\frac{2}{\left(N_{1}+N_{2}+1\right)\left(1+s^{2\left(N_{1}+N_{2}+1\right)}\right)}\right)\left(f_{1}(s)+f_{2}(s)\right) s \mathrm{~d} s .
\end{aligned}
$$


While, as a particular solution of the second equation of (4.34), we take:

$$
(\phi-\psi)(r)=(\ln r) \int_{0}^{r} t\left(f_{1}(t)-f_{2}(t)\right) \mathrm{d} t-\int_{0}^{r} t(\ln t)\left(f_{1}(t)-f_{2}(t)\right) \mathrm{d} t
$$

and we easily check that $(\phi-\psi)(r)$ is well defined and moreover $(\phi-\psi)(r) \in Y_{\alpha}^{r}$.

At this point, we readily derive $(\phi, \psi) \in Y_{\alpha}^{r} \times Y_{\alpha}^{r}$ which provides a solution for (4.34).

By the expression of $u_{0}, v_{0}$ in (4.6), we see that $\mathrm{e}^{u_{0}}$ and $\mathrm{e}^{v_{0}}$ belong to $X_{\alpha}^{r}$. Therefore, by means of the arguments above, we obtain a radial solution of (4.11) as follows:

$$
\begin{aligned}
u_{2}(r)= & \frac{1}{2}\left(\phi_{0}(r) \ln r+\frac{2}{\left(N_{1}+N_{2}+1\right)\left(1+r^{2\left(N_{1}+N_{2}+1\right)}\right)}\right) \int_{0}^{r} \phi_{0}(t)\left(\mathrm{e}^{u_{0}(t)}-\mathrm{e}^{v_{0}(t)}\right) t \mathrm{~d} t \\
& -\frac{1}{2} \phi_{0}(r) \int_{0}^{r}\left(\phi_{0}(t) \ln t+\frac{2}{\left(N_{1}+N_{2}+1\right)\left(1+s^{2\left(N_{1}+N_{2}+1\right)}\right)}\right)\left(\mathrm{e}^{u_{0}(t)}-\mathrm{e}^{v_{0}(t)}\right) t \mathrm{~d} t \\
& -\frac{1}{2}(\ln r) \int_{0}^{r} t\left(\mathrm{e}^{u_{0}(t)}+\mathrm{e}^{v_{0}(t)}\right) t \mathrm{~d} t+\frac{1}{2} \int_{0}^{r} t(\ln t)\left(\mathrm{e}^{u_{0}(t)}+\mathrm{e}^{v_{0}(t)}\right) \mathrm{d} t, \\
v_{2}(r)= & \frac{1}{2}\left(\phi_{0}(r) \ln r+\frac{2}{\left(N_{1}+N_{2}+1\right)\left(1+r^{2\left(N_{1}+N_{2}+1\right)}\right)} \int_{0}^{r}(t)\left(\mathrm{e}^{u_{0}(t)}-\mathrm{e}^{v_{0}(t)}\right) t \mathrm{~d} t\right. \\
& -\frac{1}{2} \phi_{0}(r) \int_{0}^{r}\left(\phi_{0}(t) \ln t+\frac{2}{\left(N_{1}+N_{2}+1\right)\left(1+s^{2\left(N_{1}+N_{2}+1\right)}\right)}\right)\left(\mathrm{e}^{u_{0}(t)}-\mathrm{e}^{v_{0}(t)}\right) t \mathrm{~d} t \\
& +\frac{1}{2}(\ln r) \int_{0}^{r} t\left(\mathrm{e}^{u_{0}(t)}+\mathrm{e}^{v_{0}(t)}\right) \mathrm{d} t-\frac{1}{2} \int_{0}^{r} t(\ln t)\left(\mathrm{e}^{u_{0}(t)}+\mathrm{e}^{v_{0}(t)}\right) \mathrm{d} t .
\end{aligned}
$$

Furthermore, the values of the finite integrals:

$$
\begin{aligned}
& \sigma_{1} \equiv \int_{0}^{\infty} \phi_{0}(t)\left(\mathrm{e}^{u_{0}(t)}-\mathrm{e}^{v_{0}(t)}\right) t \mathrm{~d} t \\
& \sigma_{2} \equiv \int_{0}^{\infty}\left(\mathrm{e}^{u_{0}(t)}+\mathrm{e}^{v_{0}(t)}\right) t \mathrm{~d} t
\end{aligned}
$$

characterize the asymptotic behavior for $\left(u_{2}, v_{2}\right)$ as follows:

$$
u_{2}(r)=-\frac{\sigma_{1}+\sigma_{2}}{2} \ln r+\mathrm{O}(1), \quad v_{2}(r)=-\frac{\sigma_{1}-\sigma_{2}}{2} \ln r+\mathrm{O}(1), \quad \text { as } \quad r \rightarrow \infty .
$$

Let $U_{\alpha}$ be the subspace of $Y_{\alpha}^{r} \times Y_{\alpha}^{r}$ orthogonal to $\operatorname{Ker} A$. Namely:

$$
U_{\alpha} \equiv(\operatorname{Ker} A)^{\perp} \subset Y_{\alpha}^{r} \times Y_{\alpha}^{r},
$$

we establish the following existence result:

Theorem 4.1 For fixed $\alpha \in(0,1)$, there exist $\varepsilon_{0}>0$ sufficiently small and smooth functions $\left(u_{3, \varepsilon}, v_{3, \varepsilon}\right):\left(-\varepsilon_{0}, \varepsilon_{0}\right) \rightarrow U_{\alpha}$ with $\left(u_{3,0}, v_{3,0}\right)=(0,0)$ such that, by setting:

$$
\begin{aligned}
u^{\varepsilon}(r) & \equiv u_{0}\left(\frac{r}{\varepsilon}\right)+\varepsilon u_{2}\left(\frac{r}{\varepsilon}\right)+\varepsilon u_{3, \varepsilon}\left(\frac{r}{\varepsilon}\right)+\ln \frac{1}{\varepsilon}, \\
v^{\varepsilon}(r) & \equiv v_{0}\left(\frac{r}{\varepsilon}\right)+\varepsilon v_{2}\left(\frac{r}{\varepsilon}\right)+\varepsilon v_{3, \varepsilon}\left(\frac{r}{\varepsilon}\right)+\ln \frac{1}{\varepsilon}
\end{aligned}
$$


then $\left(u^{\varepsilon}(r), v^{\varepsilon}(r)\right)$ defines a radial solution of the system (3.1) with $\left(u_{2}, v_{2}\right)$ given by (4.35) -(4.36) and satisfying the asymptotic behavior (4.39). Moreover, as $\varepsilon \rightarrow 0^{+},\left\|u_{3, \varepsilon}\right\|_{Y_{\alpha}^{r}}+\left\|v_{3, \varepsilon}\right\|_{Y_{\alpha}^{r}} \rightarrow 0$ and the following estimates hold:

$$
\begin{aligned}
& \left|u_{3, \varepsilon}(r)\right|+\left|v_{3, \varepsilon}(r)\right|=\left(\left\|u_{3, \varepsilon}\right\|_{Y_{\alpha}^{r}}+\left\|v_{3, \varepsilon}\right\|_{Y_{\alpha}^{r}}\right) \ln (1+r), \quad \forall r>0, \\
& \left|u_{3, \varepsilon}^{\prime}(r)\right|+\left|v_{3, \varepsilon}^{\prime}(r)\right|=\frac{1}{1+r}\left(\left\|u_{3, \varepsilon}\right\|_{Y_{\alpha}^{r}}+\left\|v_{3, \varepsilon}\right\|_{Y_{\alpha}^{r}}\right), \quad \forall r>0, \\
& \int_{\mathbb{R}^{2}} \mathrm{e}^{v^{\varepsilon}}\left(\mathrm{e}^{u^{\varepsilon}}+1\right) \mathrm{d} x=4 \pi\left(N_{1}+N_{2}+1\right)+\mathrm{o}(1), \\
& \int_{\mathbb{R}^{2}} \mathrm{e}^{u^{\varepsilon}}\left(\mathrm{e}^{v^{\varepsilon}}-1\right) \mathrm{d} x=4 \pi\left(N_{1}+N_{2}+1\right)+\mathrm{o}(1) .
\end{aligned}
$$

Proof. Since the linearized operator $A$ defined by (4.24) gives an isomorphism between $U_{\alpha}$ and $X_{\alpha}^{r} \times X_{\alpha}^{r}$, we can use the implicit function theorem (see e.g. [51]) for the operator $P: U_{\alpha} \times \mathbb{R} \rightarrow$ $X_{\alpha}^{r} \times X_{\alpha}^{r}$ around the point $(0,0,0)$. Therefore, for sufficiently small $\varepsilon_{0}>0$, there exists a continuous function: $\varepsilon \rightarrow \Psi_{\varepsilon} \equiv\left(u_{3, \varepsilon}, v_{3, \varepsilon}\right)$ form $\left(-\varepsilon_{0}, \varepsilon_{0}\right)$ into a neighborhood of the origin in $U_{\alpha}$ such that $P\left(u_{3, \varepsilon}, v_{3, \varepsilon}, \varepsilon\right)=0, \forall \varepsilon \in\left(-\varepsilon_{0}, \varepsilon_{0}\right)$ and $\left(u_{3,0}, v_{3,0}\right)=(0,0)$. As a consequence, $\left(u^{\varepsilon}(r), v^{\varepsilon}(r)\right)$ defined by (4.41) (4.42) defines a radial solution of the system (3.1).

In particular, $\left\|u_{3, \varepsilon}\right\|_{Y_{\alpha}^{r}}+\left\|v_{3, \varepsilon}\right\|_{Y_{\alpha}^{r}} \rightarrow 0$ as $\varepsilon \rightarrow 0^{+}$, and we can use (4.20) together with the equations (4.13), to deduce the estimates (4.43) and (4.44).

In view of (4.41)-4.42), we have:

$$
\int_{\mathbb{R}^{2}} \mathrm{e}^{v^{\varepsilon}}\left(\mathrm{e}^{u^{\varepsilon}}+1\right) \mathrm{d} x=\int_{\mathbb{R}^{2}} \mathrm{e}^{v_{0}(r)+\varepsilon v_{2}(r)+\varepsilon v_{3, \varepsilon}(r)}\left(\mathrm{e}^{u_{0}(r)+\varepsilon u_{2}(r)+\varepsilon u_{3, \varepsilon}(r)}+\varepsilon\right) \mathrm{d} x,
$$

and by the asymptotic estimates for $u_{2}, v_{2}, u_{3, \varepsilon}$, and $v_{3, \varepsilon}$ established above, we conclude that,

$$
\begin{aligned}
\lim _{\varepsilon \rightarrow 0} \int_{\mathbb{R}^{2}} \mathrm{e}^{v^{\varepsilon}}\left(\mathrm{e}^{u^{\varepsilon}}+1\right) \mathrm{d} x & =\int_{\mathbb{R}^{2}} \mathrm{e}^{u_{0}(r)+v_{0}(r)} \mathrm{d} x \\
& =8 \pi\left(N_{1}+N_{2}+1\right)^{2} \int_{0}^{\infty} \frac{r^{2\left(N_{1}+N_{2}\right)}}{\left(1+r^{2\left(N_{1}+N_{2}+1\right)}\right)^{2}} r \mathrm{~d} r \\
& =4 \pi\left(N_{1}+N_{2}+1\right),
\end{aligned}
$$

as claimed.

Similarly, we obtain:

$$
\lim _{\varepsilon \rightarrow 0} \int_{\mathbb{R}^{2}} \mathrm{e}^{u^{\varepsilon}}\left(\mathrm{e}^{v^{\varepsilon}}-1\right) \mathrm{d} x=4 \pi\left(N_{1}+N_{2}+1\right),
$$

and the proof is completed.

\section{The proof of Theorem 2.1 .}

At this point, by means of Theorem 4.1 we can establish easily Theorem 2.1,

In fact, by using (2.48) (2.49) (considered with the lower sign), together with the relations (2.67) - (2.72), we find that,

$$
\begin{aligned}
& f_{12}^{(1), \varepsilon}=\frac{8 \pi^{2}}{k^{2}}\left|\phi_{2}^{\varepsilon}\right|^{2}\left(\left|\phi_{1}^{\varepsilon}\right|^{2}+c^{2}\right)=2 \sigma^{2} \mathrm{e}^{v^{\varepsilon}}\left(\mathrm{e}^{u^{\varepsilon}}+1\right), \\
& f_{12}^{(2), \varepsilon}=\frac{8 \pi^{2}}{k^{2}}\left|\phi_{1}^{\varepsilon}\right|^{2}\left(\left|\phi_{2}^{\varepsilon}\right|^{2}-c^{2}\right)=2 \sigma^{2} \mathrm{e}^{u^{\varepsilon}}\left(\mathrm{e}^{v^{\varepsilon}}-1\right) .
\end{aligned}
$$


On the other hand, if we use the decomposition (4.41) and (4.42) for $u^{\varepsilon}$ and $v^{\varepsilon}$ together with (4.6) and the given decay estimates for the error term, we easily check that, as $\varepsilon \rightarrow 0^{+}$,

$$
\begin{aligned}
& \mathrm{e}^{u^{\varepsilon}+v^{\varepsilon}} \rightarrow 4 \pi\left(N_{1}+N_{2}+1\right) \delta_{0} \quad \text { weakly in the sense of measure in } \mathbb{R}^{2}, \\
& \mathrm{e}^{u^{\varepsilon}}, \mathrm{e}^{v^{\varepsilon}} \rightarrow 0 \text { in } L^{1}\left(\mathbb{R}^{2}\right) .
\end{aligned}
$$

Therefore, by taking into account the normalization (2.79) and (2.75), from (4.50)-(4.51), we readily deduce (2.54) and (2.55). Clearly, also (2.56) and (2.57) are a direct consequence of (4.45)(4.46). Finally, (2.53) follows from (4.6) and the given decay estimate of the error terms.

Theorem 2.1 is established.

\section{The radial problem}

In this section we collect some general properties about radial solutions of (3.1).

More precisely, for $u=u(r), v=v(r)$, we consider the following problem:

$$
\left\{\begin{array}{l}
-\left(r u^{\prime}\right)^{\prime}=r \mathrm{e}^{v}\left(\mathrm{e}^{u}+1\right), r>0 \\
-\left(r v^{\prime}\right)^{\prime}=r \mathrm{e}^{u}\left(\mathrm{e}^{v}-1\right), r>0 \\
u(r)-2 N_{1} \ln r=\mathrm{O}(1) \quad \text { as } \quad r \rightarrow 0^{+} \\
v(r)-2 N_{2} \ln r=\mathrm{O}(1) \quad \text { as } \quad r \rightarrow 0^{+}
\end{array}\right.
$$

We start to point out the following extended version of Pohozaev identity (3.4) valid in the radial setting:

Proposition 5.1 For any solution $(u, v)$ of the problem (5.1), we have:

$$
\begin{aligned}
& 2 \int_{0}^{r} t \mathrm{e}^{u(t)+v(t)} \mathrm{d} t \\
& =4\left(N_{1}+1\right)\left(N_{2}+1\right)-\left(r u^{\prime}(r)+2\right)\left(r v^{\prime}(r)+2\right)-r^{2}\left(\mathrm{e}^{u(r)+v(r)}+\mathrm{e}^{v(r)}-\mathrm{e}^{u(r)}\right), \\
& 2 \int_{0}^{r} t \mathrm{e}^{u(t)} \mathrm{d} t=4 N_{1}\left(N_{2}+1\right)-r u^{\prime}(r)\left(r v^{\prime}(r)+2\right)+r^{2}\left(\mathrm{e}^{u(r)+v(r)}+\mathrm{e}^{v(r)}-\mathrm{e}^{u(r)}\right), \\
& 2 \int_{0}^{r} t \mathrm{e}^{v(t)} \mathrm{d} t=r v^{\prime}(r)\left(r u^{\prime}(r)+2\right)-4 N_{2}\left(N_{1}+1\right)+r^{2}\left(\mathrm{e}^{u(r)+v(r)}+\mathrm{e}^{v(r)}-\mathrm{e}^{u(r)}\right) .
\end{aligned}
$$

Proof. By integration and by using (5.1) we find:

$$
\begin{aligned}
& r u^{\prime}(r)=2 N_{1}-\int_{0}^{r} t \mathrm{e}^{v(t)}\left(\mathrm{e}^{u(t)}+1\right) \mathrm{d} t \\
& r v^{\prime}(r)=2 N_{2}-\int_{0}^{r} t \mathrm{e}^{u(t)}\left(\mathrm{e}^{v(t)}-1\right) \mathrm{d} t .
\end{aligned}
$$

Multiplying the first equation in (5.1) by $r v^{\prime}$, and the second equation in (5.1) by $r u^{\prime}$, and after integrating by parts, we obtain:

$$
\begin{aligned}
& 4 N_{1} N_{2}-r^{2} u^{\prime}(r) v^{\prime}(r) \\
& =r^{2}\left(\mathrm{e}^{u(r)+v(r)}+\mathrm{e}^{v(r)}-\mathrm{e}^{u(r)}\right)-2 \int_{0}^{r} t \mathrm{e}^{u(t)+v(t)} \mathrm{d} t-2 \int_{0}^{r} t\left(\mathrm{e}^{v(t)}-\mathrm{e}^{u(t)}\right) \mathrm{d} t .
\end{aligned}
$$


Combining (5.5)-(5.7), we obtain (5.2)-(5.4).

Interestingly, for radial solutions of (5.1), the following integrability properties hold:

Lemma 5.1 Let $(u, v)$ be a solution of (5.1), then we have:

$$
\int_{0}^{\infty} r \mathrm{e}^{u+v} \mathrm{~d} r<\infty, \quad \int_{0}^{\infty} r \mathrm{e}^{u} \mathrm{~d} r<\infty
$$

Proof. We argue by contradiction and suppose $\int_{0}^{r} t \mathrm{e}^{u+v} \mathrm{~d} t \rightarrow \infty$ as $r \rightarrow \infty$. We see that $r u^{\prime}(r)$ is monotone decreasing and diverges to $-\infty$ as $r \rightarrow \infty$. As a consequence, $\frac{u(r)}{\ln r} \rightarrow-\infty$ as $r \rightarrow \infty$, and so, $\int_{0}^{\infty} r \mathrm{e}^{u} \mathrm{~d} r<\infty$. At this point we can use (5.6) to conclude that also, $\lim _{r \rightarrow \infty} \frac{v(r)}{\ln r}=-\infty$, and so: $\int_{0}^{\infty} r \mathrm{e}^{u+v} \mathrm{~d} r<\infty$, a contradiction.

Similarly, we get that, $\int_{0}^{\infty} r \mathrm{e}^{u} \mathrm{~d} r<\infty$. Indeed, if by contradiction, we suppose that, $\int_{0}^{r} t \mathrm{e}^{u} \mathrm{~d} t \rightarrow$ $\infty$ as $r \rightarrow \infty$, then from (5.6), we find that $r v^{\prime} \rightarrow \infty$ as $r \rightarrow \infty$. As a consequence, $\int_{0}^{r} t \mathrm{e}^{v} \mathrm{~d} t \rightarrow \infty$ as $r \rightarrow \infty$ and in view of (5.5), we have $r u^{\prime} \rightarrow-\infty$ as $r \rightarrow \infty$. In other words, $\frac{u(r)}{\ln r} \rightarrow-\infty$ and so $\int_{0}^{\infty} r \mathrm{e}^{u} \mathrm{~d} r<\infty$, a contradiction.

In view of Lemma 5.1, to obtain a (radial) solution of (3.1), we only need to ensure the integrability of $\mathrm{e}^{v}$. To this purpose, let

$$
u(r)=U(r)+2 N_{1} \ln r, \quad v(r)=V(r)+2 N_{2} \ln r .
$$

From now on we use the following notation:

$$
\left\{\begin{array}{l}
F_{1}(r) \equiv \int_{0}^{r} t^{2 N_{2}+1} \mathrm{e}^{V(t)}\left(t^{2 N_{1}} \mathrm{e}^{U(t)}+1\right) \mathrm{d} t=\int_{0}^{r} t \mathrm{e}^{v(t)}\left(\mathrm{e}^{u(t)}+1\right) \mathrm{d} t \\
F_{2}(r) \equiv \int_{0}^{r} t^{2 N_{1}+1} \mathrm{e}^{U(t)}\left(t^{2 N_{2}} \mathrm{e}^{V(t)}-1\right) \mathrm{d} t=\int_{0}^{r} t \mathrm{e}^{u(t)}\left(\mathrm{e}^{v(t)}-1\right) \mathrm{d} t
\end{array}\right.
$$

and we consider the initial value problem for the (regular) functions $(U(r), V(r))$ associated to (5.1). Namely:

$$
\left\{\begin{array}{l}
-\left(r U^{\prime}\right)^{\prime}=r^{2 N_{2}+1} \mathrm{e}^{V}\left(r^{2 N_{1}} \mathrm{e}^{U}+1\right), \\
-\left(r V^{\prime}\right)^{\prime}=r^{2 N_{1}+1} \mathrm{e}^{U}\left(r^{2 N_{2}} \mathrm{e}^{V}-1\right), \\
U(0)=\alpha_{1}, V(0)=\alpha_{2}, \\
U^{\prime}(0)=V^{\prime}(0)=0,
\end{array}\right.
$$

with $\left(\alpha_{1}, \alpha_{2}\right) \in \mathbb{R}^{2}$.

For the initial value problem (5.11), the following holds:

Proposition 5.2 For any $\left(\alpha_{1}, \alpha_{2}\right) \in \mathbb{R}^{2}$, the initial value problem (5.11) admits a unique global solution, which depends continuously on the initial data. 
Proof. By standard ODE techniques, we see that the system (5.11) admits a unique local solution $(U(r), V(r))$ defined in the interval $\left(0, r_{0}\right)$ for some $r_{0}>0$. By integration, for every $r \in\left(0, r_{0}\right)$ we have:

$$
r U^{\prime}(r)=-F_{1}(r)<0 \text { and } r V^{\prime}(t)=-F_{2}(r) .
$$

In particular, we see that $U(r)$ is strictly decreasing in $\left(0, r_{0}\right)$, and so $U(r) \leq U(0)=\alpha_{1}$, for $0<r<r_{0}$. While, by (5.12), for $0<r<r_{0}$ we get,

$$
V(r)=V(0)-\int_{0}^{r} \frac{1}{t} F_{2}(t) \mathrm{d} t \leq V(0)+\int_{0}^{r} \frac{1}{t} \int_{0}^{t} s^{2 N_{1}+1} \mathrm{e}^{U(s)} \mathrm{d} s \mathrm{~d} t \leq V(0)+\frac{\mathrm{e}^{U(0)} r_{0}^{2\left(N_{1}+1\right)}}{4\left(N_{1}+1\right)^{2}} .
$$

By (5.12), we see that, for every $0<r<r_{0}$ the following holds:

$$
\left|U^{\prime}(r)\right| \leq\left|\frac{1}{r} F_{1}(r)\right| \leq C \text { and } \quad\left|V^{\prime}(r)\right| \leq\left|\frac{1}{r} F_{2}(r)\right| \leq C .
$$

for some constant $C>0$ depending only on the initial data $\left(\alpha_{1}, \alpha_{2}\right)$.

Hence, for all $0 \leq r<r_{0}$, we have:

$$
|U(r)| \leq|U(0)|+C r_{0} \quad \text { and } \quad|V(r)| \leq|V(0)|+C r_{0} .
$$

At this point, we can use a standard unique continuation argument to conclude that the initial value problem (5.11) admits a unique global solution, which depends continuously on the initial data $\left(\alpha_{1}, \alpha_{2}\right) \in \mathbb{R}^{2}$.

Remark 5.1 In view of Proposition 5.2, we know that every solution of the radial problem (5.1) corresponds (via (5.9) ) to a solution of the initial value problem (5.11) and viceversa, in other words, problem (5.1) and (5.11) are equivalent.

Furthermore, from (5.12) we see that, in case $F_{1}(\infty)<\infty$, then the following estimate holds:

$$
U(r)>-F_{1}(\infty) \ln r-C, \forall r \geq 1,
$$

with $C>0$ a suitable constant, and it implies that necessarily $F_{1}(\infty)>2\left(N_{1}+1\right)$ (as already pointed out in Section (3). With this information, we can show in turn that a similar estimate holds for $U+V$, namely:

$$
U(r)+V(r) \geq-\left(F_{1}(\infty)+F_{2}(\infty)\right) \ln r-C, \quad \forall r \geq 1,
$$

and by Lemma 5.1, we conclude that: $F_{1}(\infty)+F_{2}(\infty)>2\left(N_{1}+N_{2}+1\right)$. As a consequence,

$$
r^{2\left(N_{1}+N_{2}+1\right)} \mathrm{e}^{U(r)+V(r)} \rightarrow 0, \quad r^{2\left(N_{1}+1\right)} \mathrm{e}^{U(r)} \rightarrow 0 \quad \text { as } r \rightarrow \infty .
$$

To proceed further, we point out some qualitative information about solutions of (5.1). First of all, from (5.9) and (5.17), we see that $u(r) \rightarrow-\infty$ as $r \rightarrow 0^{+}$and $u(r)+2 \ln r \rightarrow-\infty$ as $r \rightarrow \infty$, so both $u(r)$ and $u(r)+2 \ln r$ admit a unique maximum attained respectively at the (unique) values: $0<t_{1}<\tilde{t}_{1}$ satisfying: $F_{1}\left(t_{1}\right)=2 N_{1}$ and $F_{1}\left(\tilde{t}_{1}\right)=2\left(N_{1}+1\right)$, see (5.5). Furthermore, we can also conclude that, in case the maximum value of $u(r)$ (or $u(r)+2 \ln r$ ) is positive then $u(r)$ (or $u(r)+2 \ln r)$ must vanish exactly twice.

Less obvious is the behavior of $v(r)$, and we can describe it in terms of the value of $F_{2}(\infty)$ as follows: 
Proposition 5.3 Let $(u, v)$ satisfy (5.1). There holds:

(i) $v(r)$ vanishes at least once at a value $r_{0}>0$ which corresponds to the global minimum point of $F_{2}(r)$. Furthermore, we have that, $v(r)<0, \forall r \in\left(0, r_{0}\right)$.

(ii) $F_{2}(r)$ can attain the value $2 N_{2}$ at most once and:

a) If $F_{2}(\infty)<2 N_{2}$, then $F_{2}(r)<2 N_{2}, \forall r>0$, $v$ is strictly increasing (to $\infty$ ) with unique zero at $r_{0}$, and so $F_{2}(r)$ is strictly decreasing in $\left(0, r_{0}\right)$ and strictly increasing in $\left[r_{0}, \infty\right)$;

b) If $F_{2}(\infty)>2 N_{2}$, then $v$ vanishes exactly twice at $r_{0}<t_{0}$, and $r_{0}$ corresponds to the global minimum point of $F_{2}(r)$ while $t_{0}$ corresponds to the global maximum point of $F_{2}(r)$. Moreover, $F_{2}$ is decreasing in $\left[0, r_{0}\right] \cup\left[t_{0}, \infty\right)$ and increasing in $\left(r_{0}, t_{0}\right)$. Furthermore, $v$ admits a unique maximum at $r_{*} \in\left(r_{0}, t_{0}\right)$ satisfying $F_{2}\left(r_{*}\right)=2 N_{2}$.

Proof. To prove (i), we first observe that $v(r)<0$ for $r$ sufficiently close to $r=0$. So the set $\Lambda \equiv\{r>0 \mid v(t) \leq 0, \forall t \in(0, r]\}$ is not empty.

Claim 1: $\Lambda$ is bounded from above and $r_{0}=\sup \Lambda$.

To establish Claim 1, we argue by contradiction and assume that $v(r) \leq 0, \forall r>0$. As a consequence, $F_{2}(r)<0, \forall r>0$ and so the function $V(r)=v(r)-2 N_{2} \ln r$ is strictly increasing and must satisfy: $r^{2 N_{2}} \mathrm{e}^{V(r)}=\mathrm{e}^{v(r)} \leq 1, \forall r>0$. In particular, $r^{2 N_{2}} \mathrm{e}^{V(0)} \leq 1$ for all $r>0$, and this is clearly impossible.

At this point, if we set $r_{0}=\sup \Lambda$, we find that: $v\left(r_{0}\right)=0$ and $F_{2}\left(r_{0}\right)<0$. So, for $\delta>0$ sufficiently small we still have $F_{2}(r)<0, \forall r \in\left[0, r_{0}+\delta\right]$. Thus, $v$ is increasing in $\left(0, r_{0}+\delta\right)$ and in particular $v(t)<0, \forall t \in\left(0, r_{0}\right)$, while $v(r)>0$ as $r \in\left(r_{0}, r_{0}+\delta\right)$. By those information we conclude the proof of Claim 1 and also see that $r_{0}$ defines a strictly local minimum for $F_{2}(r)$. We shall show below that actually $r_{0}$ is a global minimum for $F_{2}(r)$.

But first we establish (ii). We see that, $F_{2}(r)<0$ for $r \in\left(0, r_{0}\right]$ and so we consider the set

$$
\Lambda_{1}=\left\{r>0 \mid \forall t \in(0, r): F_{2}(t)<2 N_{2}\right\} \supset\left(0, r_{0}\right] .
$$

In case $\Lambda_{1}$ is unbounded above, we see that in this case $F_{2}(r)$ never attains the value $2 N_{2}$. Hence we assume that, $\tau_{1}=\sup \Lambda_{1}<\infty$. Consequently, $F_{2}\left(\tau_{1}\right)=2 N_{2}$ and $F_{2}(r)<2 N_{2}, \forall r \in\left[0, \tau_{1}\right)$. Furthermore, we know that $\tau_{1}>r_{0}$ and $v$ is strictly increasing in $\left[0, \tau_{1}\right]$. As a consequence, $v\left(\tau_{1}\right)>0\left(=v\left(r_{0}\right)\right)$.

Claim 2: $F_{2}(r)>2 N_{2}, \forall r>\tau_{1}$.

To establish Claim 2, we observe first that, for $\varepsilon>0$ sufficiently small, we have: $v(r)>0, \forall r \in$ $\left[\tau_{1}, \tau_{1}+\varepsilon\right]$, and so $F_{2}(r)>2 N_{2}, \forall r \in\left(\tau_{1}, \tau_{1}+\varepsilon\right]$. We argue again by contradiction and assume that,

$$
\tau_{2}=\sup \left\{r>\tau_{1}, \forall t \in\left(\tau_{1}, r\right) \mid F_{2}(r)>2 N_{2}\right\}<\infty .
$$

Hence, $\tau_{2}>\tau_{1}, F_{2}\left(\tau_{2}\right)=2 N_{2}$ and $F_{2}(r)>2 N_{2}, \forall r \in\left(\tau_{1}, \tau_{2}\right)$. In particular, $v(r)$ is strictly decreasing in $\left(\tau_{1}, \tau_{2}\right)$ and $v\left(\tau_{2}\right)<0$. In other words, we have obtained the following:

$$
\begin{aligned}
& 0<\tau_{1}<\tau_{2}: F_{2}\left(\tau_{1}\right)=F_{2}\left(\tau_{2}\right)=2 N_{2} \text { and } v\left(\tau_{2}\right)<0<v\left(\tau_{1}\right), \\
& v\left(\tau_{2}\right)<v(\tau)<v\left(\tau_{1}\right), \forall r \in\left(\tau_{1}, \tau_{2}\right) .
\end{aligned}
$$

We show next that (5.18)-(5.19) are impossible (i.e. $v$ cannot "oscillate"). Indeed, by using 
Pohozaev identity (5.2) together with (5.5) and (5.6) respectively at $r=\tau_{1}$ and $r=\tau_{2}$, we have:

$$
\begin{aligned}
& {\left[2\left(N_{1}+1\right)-F_{1}\left(\tau_{1}\right)\right]\left[2\left(N_{2}+1\right)-F_{2}\left(\tau_{1}\right)\right]+2 \int_{0}^{\tau_{1}} t \mathrm{e}^{u(t)+v(t)} \mathrm{d} t+\tau_{1}^{2}\left(\mathrm{e}^{u\left(\tau_{1}\right)+v\left(\tau_{1}\right)}+\mathrm{e}^{v\left(\tau_{1}\right)}-\mathrm{e}^{u\left(\tau_{1}\right)}\right) } \\
= & {\left[2\left(N_{1}+1\right)-F_{1}\left(\tau_{2}\right)\right]\left[2\left(N_{2}+1\right)-F_{2}\left(\tau_{2}\right)\right]+2 \int_{0}^{\tau_{2}} t \mathrm{e}^{u(t)+v(t)} \mathrm{d} t+\tau_{2}^{2}\left(\mathrm{e}^{u\left(\tau_{2}\right)+v\left(\tau_{2}\right)}+\mathrm{e}^{v\left(\tau_{2}\right)}-\mathrm{e}^{u\left(\tau_{2}\right)}\right), }
\end{aligned}
$$

and since $F_{2}\left(\tau_{1}\right)=F_{2}\left(\tau_{2}\right)=2 N_{2}$, we obtain:

$-2 \int_{0}^{\tau_{1}} t \mathrm{e}^{v(t)} \mathrm{d} t+\tau_{1}^{2}\left(\mathrm{e}^{u\left(\tau_{1}\right)+v\left(\tau_{1}\right)}+\mathrm{e}^{v\left(\tau_{1}\right)}-\mathrm{e}^{u\left(\tau_{1}\right)}\right)=-2 \int_{0}^{\tau_{2}} t \mathrm{e}^{v(t)} \mathrm{d} t+\tau_{2}^{2}\left(\mathrm{e}^{u\left(\tau_{2}\right)+v\left(\tau_{2}\right)}+\mathrm{e}^{v\left(\tau_{2}\right)}-\mathrm{e}^{u\left(\tau_{2}\right)}\right)$.

Consequently, since $v\left(\tau_{2}\right)<0<v\left(\tau_{1}\right)$, we derive:

$$
\begin{aligned}
2 \int_{\tau_{1}}^{\tau_{2}} t \mathrm{e}^{v(t)} \mathrm{d} t & =\tau_{2}^{2} \mathrm{e}^{u\left(\tau_{2}\right)}\left(\mathrm{e}^{v\left(\tau_{2}\right)}-1\right)+\tau_{2}^{2} \mathrm{e}^{v\left(\tau_{2}\right)}-\tau_{1}^{2} \mathrm{e}^{u\left(\tau_{1}\right)}\left(\mathrm{e}^{v\left(\tau_{1}\right)}-1\right)-\tau_{1}^{2} \mathrm{e}^{v\left(\tau_{1}\right)} \\
& <\tau_{2}^{2} \mathrm{e}^{v\left(\tau_{2}\right)}-\tau_{1}^{2} \mathrm{e}^{v\left(\tau_{1}\right)} .
\end{aligned}
$$

On the other hand, $v$ is strictly decreasing in $\left[\tau_{1}, \tau_{2}\right]$, and so we have:

$$
\left(\tau_{2}^{2}-\tau_{1}^{2}\right) \mathrm{e}^{v\left(\tau_{2}\right)}<2 \int_{\tau_{1}}^{\tau_{2}} t \mathrm{e}^{v(t)} \mathrm{d} t \leq \tau_{2}^{2} \mathrm{e}^{v\left(\tau_{2}\right)}-\tau_{1}^{2} \mathrm{e}^{v\left(\tau_{1}\right)}
$$

which implies that: $\tau_{1}^{2}\left(\mathrm{e}^{v\left(\tau_{1}\right)}-\mathrm{e}^{v\left(\tau_{2}\right)}\right)<0$, a contradiction to the fact that: $v\left(\tau_{1}\right)>v\left(\tau_{2}\right)$. Therefore, Claim 2 is established, as well as (ii).

If $F_{2}(\infty)<2 N_{2}$, by virtue of (ii) we see that necessarily: $F_{2}(r)<2 N_{2}, \forall r>0$. Hence $v$ is strictly increasing and $v(r) \rightarrow \infty$ as $r \rightarrow \infty$, and so, $r_{0}$ is the only zero of $v$. So, $F_{2}(r)$ is strictly decreasing in $\left(0, r_{0}\right)$ and strictly increasing in $\left(r_{0}, \infty\right)$, and consequently $r_{0}$ corresponds to its global minimum point. On the other hand, if $F_{2}(\infty)>2 N_{2}$, then by (ii), we know that there exists a unique value $r_{*}>0$ such that, $F_{2}(r)<2 N_{2}, \forall r \in\left(0, r_{*}\right)$ and $F_{2}(r)>2 N_{2}$ for $r>r_{*}$ (see Claim 2) and $r_{*}>r_{0}$ (see Claim 1). Consequently, $v$ is strictly increasing in $\left(0, r_{*}\right)$ and strictly decreasing in $\left(r_{*}, \infty\right)$ and $v$ attains its maximum value at $r_{*}$. Furthermore: $\frac{v(r)}{\ln r} \rightarrow\left(2 N_{2}-F_{2}(\infty)\right)<0$ as $r \rightarrow \infty$, so we see that $v(r) \rightarrow-\infty$ as $r \rightarrow \infty$, and so $v$ must vanish once at $t_{0} \in\left(r_{*}, \infty\right)$. This implies that $F_{2}(r)$ is strictly decreasing in $\left(0, r_{0}\right] \cup\left[t_{0}, \infty\right)$, while it is increasing in $\left(r_{0}, t_{0}\right)$, so $r_{0}$ and $t_{0}$ corresponds respectively to the global minimum and global maximum points for $F_{2}(r)$.

The case $F_{2}(\infty)=2 N_{2}$ enters as a border line case, and for $v(r)$ and $F_{2}(r)$ both behaviors a) and b) could occur. However, in any case, $r_{0}$ would always correspond to a global minimum point for $F_{2}(r)$, and the proof is completed.

From Proposition 5.3 we can derive easily some useful information about $(U, V)$ solutions of (5.11). Clearly, $U$ is strictly decreasing (to $-\infty$ ), namely: $U(r) \leq U(0), \forall r>0$. Concerning $V$, we have:

Corollary 5.1 Let $(U, V)$ be a solution of (5.11), we have:

(i) If $F_{2}(\infty) \leq 0$, then $F_{2}(r)<0, \forall r>0$ and $V$ is strictly increasing $($ to $\infty)$;

(ii) If $F_{2}(\infty)>0$, then there exists a unique $s_{0}>r_{0}$ such that $F_{2}\left(s_{0}\right)=0$, and $F_{2}(s)<0, \forall s \in$ $\left(0, s_{0}\right)$, while $F_{2}(s)>0$ for $s>s_{0}$. In particular, $s_{0}$ is the (unique) maximum point of $V$. 
Our next goal is to provide the following a priori estimates.

Proposition 5.4 For a solution $(U, V)$ of (5.11), the following estimates hold:

$$
\begin{aligned}
& \text { i) } \frac{1}{2\left(N_{1}+1\right)}\left(\max _{r \in[0, \infty)} r^{2\left(N_{1}+1\right)} \mathrm{e}^{U(r)}\right) \leq \int_{0}^{\infty} t^{2 N_{1}+1} \mathrm{e}^{U(t)} \mathrm{d} t \\
& \leq 2\left[2\left(N_{1}+1\right)+\mathrm{e}^{U(0)-\frac{N_{1}+1}{N_{2}} V(0)}\right], \\
& \text { ii) } F_{2}(r)<2\left(N_{1}+N_{2}+1\right) \text {, } \\
& \left(F_{1}(r)-2\left(N_{1}+N_{2}+1\right)\right) \int_{0}^{r} t^{2\left(N_{1}+N_{2}\right)+1} \mathrm{e}^{U(t)+V(t)} \mathrm{d} t+r^{2\left(N_{1}+N_{2}+1\right)} \mathrm{e}^{U(r)+V(r)}>0,
\end{aligned}
$$

$\forall r \geq 0 ;$ and in particular,

$$
\begin{aligned}
& \int_{0}^{\infty} t^{2\left(N_{1}+N_{2}\right)+1} \mathrm{e}^{U(t)+V(t)} \mathrm{d} t \leq 2\left[3\left(N_{1}+1\right)+N_{2}+\mathrm{e}^{U(0)-\frac{N_{1}+1}{N_{2}} V(0)}\right], \\
& \max _{r \in[0, \infty)}\left(r^{2\left(N_{1}+N_{2}+1\right)} \mathrm{e}^{U(r)+V(r)}\right) \leq\left[3\left(N_{1}+1\right)+N_{2}+\mathrm{e}^{U(0)-\frac{N_{1}+1}{N_{2}} V(0)}\right]^{2} .
\end{aligned}
$$

Proof. Recall that, $U(r) \leq U(0)$ and $V(r) \geq V(0), \forall r \in\left[0, r_{0}\right]$, with $r_{0}>0$ as given by part (i) of Proposition 5.3. Recall also that,

$$
F_{2}\left(r_{0}\right)<0 \quad \text { and } \quad F_{2}(r) \geq F_{2}\left(r_{0}\right), \forall r \geq 0 .
$$

Therefore, by means of such information and Jensen's inequality, we have:

$$
\begin{aligned}
\int_{0}^{r_{0}} t^{2 N_{1}+1} \mathrm{e}^{U(t)} \mathrm{d} t & \geq \int_{0}^{r_{0}} t^{2\left(N_{1}+N_{2}\right)+1} \mathrm{e}^{U(t)+V(t)} \mathrm{d} t \\
& =\int_{0}^{r_{0}} t^{2\left(N_{1}+N_{2}\right)+1} \mathrm{e}^{\frac{2\left(N_{1}+N_{2}\right)+1}{2 N_{1}+1} U(t)} \mathrm{e}^{V(t)-\frac{2 N_{2}}{2 N_{1}+1} U(t)} \mathrm{d} t \\
& \geq r_{0} \mathrm{e}^{V(0)-\frac{2 N_{2}}{2 N_{1}+1} U(0)} \int_{0}^{r_{0}}\left(t^{2 N_{1}+1} \mathrm{e}^{U(t)}\right)^{\frac{2\left(N_{1}+N_{2}\right)+1}{2 N_{1}+1}} \mathrm{~d} t \\
& \geq r_{0} \mathrm{e}^{V(0)-\frac{2 N_{2}}{2 N_{1}+1} U(0)}\left(f_{0}^{r_{0}} t^{2 N_{1}+1} \mathrm{e}^{U(t)} \mathrm{d} t\right)^{\frac{2\left(N_{1}+N_{2}\right)+1}{2 N_{1}+1}},
\end{aligned}
$$

which implies

$$
\int_{0}^{r_{0}} t^{2 N_{1}+1} \mathrm{e}^{U(t)} \mathrm{d} t \leq r_{0} \mathrm{e}^{U(0)-\frac{2 N_{1}+1}{2 N_{2}} V(0)}
$$

By recalling that, $0=v\left(r_{0}\right)=V\left(r_{0}\right)+2 N_{2} \ln r_{0}>V(0)+2 N_{2} \ln r_{0}$, we see that $r_{0}<\mathrm{e}^{-\frac{V(0)}{2 N_{2}}}$ and from (5.27) we obtain the following:

$$
\int_{0}^{r_{0}} t^{2 N_{1}+1} \mathrm{e}^{U(t)} \mathrm{d} t \leq \mathrm{e}^{U(0)-\frac{N_{1}+1}{N_{2}} V(0)} .
$$

Clearly, from (5.28) we find:

$$
\left|F_{2}\left(r_{0}\right)\right| \leq \int_{0}^{r_{0}} t^{2 N_{1}+1} \mathrm{e}^{U(t)} \mathrm{d} t \leq \mathrm{e}^{U(0)-\frac{N_{1}+1}{N_{2}} V(0)} .
$$


To prove (5.21), we use the first equation in (5.11) and integration by parts, to find:

$$
\begin{aligned}
& 2\left(N_{1}+1\right) \int_{0}^{r} t^{2 N_{1}+1} \mathrm{e}^{U(t)} \mathrm{d} t \\
&=r^{2\left(N_{1}+1\right)} \mathrm{e}^{U(r)}+\int_{0}^{r} t^{2 N_{1}+1} \mathrm{e}^{U(t)} F_{1}(t) \mathrm{d} t \\
&=r^{2\left(N_{1}+1\right)} \mathrm{e}^{U(r)}+\int_{0}^{r} t^{2 N_{1}+1} \mathrm{e}^{U(t)}\left(\int_{0}^{t} s^{2\left(N_{1}+N_{2}\right)+1} \mathrm{e}^{U(s)+V(s)} \mathrm{d} s-\int_{0}^{t} s^{2 N_{1}+1} \mathrm{e}^{U(s)} \mathrm{d} s\right) \\
&+\frac{1}{2}\left(\int_{0}^{r} t^{2 N_{1}+1} \mathrm{e}^{U(t)} \mathrm{d} t\right)^{2}+\int_{0}^{r} t^{2\left(N_{1}+N_{2}\right)+1} \mathrm{e}^{U(t)+V(t)} \int_{0}^{t} s \mathrm{e}^{v(s)} \mathrm{d} s \mathrm{~d} t \\
& \geq r^{2\left(N_{1}+1\right)} \mathrm{e}^{U(r)}+\int_{0}^{r} t^{2 N_{1}+1} \mathrm{e}^{U(t)} F_{2}(t) \mathrm{d} t+\frac{1}{2}\left(\int_{0}^{r} t^{2 N_{1}+1} \mathrm{e}^{U(t)} \mathrm{d} t\right)^{2} \\
& \geq r^{2\left(N_{1}+1\right)} \mathrm{e}^{U(r)}+F_{2}\left(r_{0}\right) \int_{0}^{r} t^{2 N_{1}+1} \mathrm{e}^{U(t)} \mathrm{d} t+\frac{1}{2}\left(\int_{0}^{r} t^{2 N_{1}+1} \mathrm{e}^{U(t)} \mathrm{d} t\right)^{2},
\end{aligned}
$$

which implies:

$$
\frac{r^{2\left(N_{1}+1\right)} \mathrm{e}^{U(r)}}{2\left(N_{1}+1\right)} \leq \int_{0}^{r} t^{2 N_{1}+1} \mathrm{e}^{U(t)} \mathrm{d} t \leq 2\left[2\left(N_{1}+1\right)+\left|F_{2}\left(r_{0}\right)\right|\right], \quad \forall r>0 .
$$

Thus, we can use (5.29) into (5.32), and by letting $r \rightarrow \infty$, we obtain (5.21).

Similarly, we can prove (5.24). Indeed, by simple integration by parts, we have:

$$
\begin{aligned}
2\left(N_{1}+N_{2}+1\right) \int_{0}^{r} t^{2\left(N_{1}+N_{2}\right)+1} \mathrm{e}^{U(t)+V(t)} \mathrm{d} t \\
=r^{2\left(N_{1}+N_{2}+1\right)} \mathrm{e}^{U(r)+V(r)}+\int_{0}^{r} t^{2\left(N_{1}+N_{2}\right)+1} \mathrm{e}^{U(t)+V(t)}\left(F_{1}(t)+F_{2}(t)\right) \mathrm{d} t \\
=r^{2\left(N_{1}+N_{2}+1\right)} \mathrm{e}^{U(r)+V(r)}+\left(\int_{0}^{r} t^{2\left(N_{1}+N_{2}\right)+1} \mathrm{e}^{U(t)+V(t)} \mathrm{d} t\right)^{2} \\
\quad+\int_{0}^{r} t^{2\left(N_{1}+N_{2}\right)+1} \mathrm{e}^{U(t)+V(t)}\left(\int_{0}^{t}\left(s^{2 N_{2}+1} \mathrm{e}^{V(s)}-s^{2 N_{1}+1} \mathrm{e}^{U(s)}\right) \mathrm{d} s\right) \mathrm{d} t .
\end{aligned}
$$

As a consequence,

$$
\begin{aligned}
& 2\left(N_{1}+N_{2}+1\right) \int_{0}^{r} t^{2\left(N_{1}+N_{2}\right)+1} \mathrm{e}^{U(t)+V(t)} \mathrm{d} t \\
& \geq r^{2\left(N_{1}+N_{2}+1\right)} \mathrm{e}^{U(r)+V(r)}+\left(\int_{0}^{r} t^{2\left(N_{1}+N_{2}\right)+1} \mathrm{e}^{U(t)+V(t)} \mathrm{d} t\right)^{2} \\
& \quad-\int_{0}^{r} t^{2\left(N_{1}+N_{2}\right)+1} \mathrm{e}^{U(t)+V(t)} \mathrm{d} t \int_{0}^{r} t^{2 N_{1}+1} \mathrm{e}^{U(t)} \mathrm{d} t
\end{aligned}
$$

and

$$
\begin{aligned}
& 2\left(N_{1}+N_{2}+1\right) \int_{0}^{r} t^{2\left(N_{1}+N_{2}\right)+1} \mathrm{e}^{U(t)+V(t)} \mathrm{d} t<r^{2\left(N_{1}+N_{2}+1\right)} \mathrm{e}^{U(r)+V(r)} \\
& \quad+\left(\int_{0}^{r} t^{2\left(N_{1}+N_{2}\right)+1} \mathrm{e}^{U(t)+V(t)} \mathrm{d} t\right)^{2}+\int_{0}^{r} t^{2\left(N_{1}+N_{2}\right)+1} \mathrm{e}^{U(t)+V(t)} \int_{0}^{r} t^{2 N_{2}+1} \mathrm{e}^{V(t)} \mathrm{d} t .
\end{aligned}
$$


From (5.34) we readily derive the inequality claimed in (5.23). On the other hand, by setting

$$
A(r) \equiv \int_{0}^{r} t^{2\left(N_{1}+N_{2}\right)+1} \mathrm{e}^{U(t)+V(t)} \mathrm{d} t
$$

from (5.33) we get the following inequality:

$$
\begin{aligned}
& {\left[A(r)-\left(N_{1}+N_{2}+1+\frac{1}{2} \int_{0}^{r} s^{2 N_{1}+1} \mathrm{e}^{U(s)} \mathrm{d} s\right)\right]^{2}} \\
& \leq\left(N_{1}+N_{2}+1+\frac{1}{2} \int_{0}^{r} s^{2 N_{1}+1} \mathrm{e}^{U(s)} \mathrm{d} s\right)^{2}-r^{2\left(N_{1}+N_{2}+1\right)} \mathrm{e}^{U(r)+V(r)} .
\end{aligned}
$$

Consequently, from (5.36), we obtain, $\forall r>0$ :

$$
r^{2\left(N_{1}+N_{2}+1\right)} \mathrm{e}^{U(r)+V(r)} \leq\left(N_{1}+N_{2}+1+\frac{1}{2} \int_{0}^{r} s^{2 N_{1}+1} \mathrm{e}^{U(s)} \mathrm{d} s\right)^{2}
$$

and

$$
\int_{0}^{r} t^{2\left(N_{1}+N_{2}\right)+1} \mathrm{e}^{U(t)+V(t)} \mathrm{d} t<2\left(N_{1}+N_{2}+1+\frac{1}{2} \int_{0}^{r} s^{2 N_{1}+1} \mathrm{e}^{U(s)} \mathrm{d} s\right) .
$$

At this point, from (5.38) we readily derive (5.22). Furthermore, we may also conclude the desired estimates (5.24)-(5.25), by letting $r \rightarrow \infty$ and by using (5.21).

Remark 5.2 Note in particular that from (5.22) and (5.17) we obtain the necessary condition:

$$
F_{2}(\infty)<2\left(N_{1}+N_{2}+1\right)<F_{1}(\infty) \leq \infty
$$

already derived for general finite-energy solutions (i.e. not necessarily radial) in Section 3.

\section{Existence of radial solutions and the proof of Theorem 2.2 and Theorem 2.3}

In this section we focus our attention to the radial solvability of (3.1) and carry out the proof of Theorem 2.2 and Theorem 2.3

To this end, by Lemma 5.1 and via (5.9), we need to identify the initial conditions for the Cauchy problem (5.11) which yields to a solution satisfying:

$$
\int_{0}^{\infty} t \mathrm{e}^{v(t)} \mathrm{d} t=\int_{0}^{\infty} t^{2 N_{2}+1} \mathrm{e}^{V(t)} \mathrm{d} t<\infty .
$$

According to the discussion in Section 3 (valid for solutions not necessarily radially symmetric), we know that,

Lemma 6.1 A solution of (5.11) satisfies (6.1) if and only if

$$
F_{2}(\infty)>2\left(N_{2}+1\right)
$$


Actually, in the radial case, the statement of Lemma 6.1 can be easily checked.

To this purpose, we recall that, as $r \rightarrow \infty$,

$$
\frac{V(r)}{\ln r} \rightarrow-F_{2}(\infty) \text { and } \quad \frac{U(r)}{\ln r} \rightarrow-F_{1}(\infty)<-2\left(N_{1}+N_{2}+1\right),
$$

(see Remark 5.2). While it is clear that (6.2) implies (6.1), on the contrary if (6.1) holds then we can conclude only that: $F_{2}(\infty) \geq 2\left(N_{2}+1\right)$. In particular, if (6.1) holds, then,

$$
\frac{U(r)+V(r)}{\ln r} \rightarrow-\left(F_{1}(\infty)+F_{2}(\infty)\right)<-2\left(N_{1}+N_{2}+1\right)-2\left(N_{2}+1\right), \quad \text { as } \quad r \rightarrow \infty .
$$

To check that actually, if $F_{2}(\infty)=2\left(N_{2}+1\right)$ then (6.1) fails, we use (6.3) and (6.4), to see that

$$
V(r)+2\left(N_{2}+1\right) \ln r=V(0)+\left(F_{2}(\infty)-F_{2}(r)\right) \ln r+\int_{0}^{r}(\ln t) F_{2}^{\prime}(t) \mathrm{d} t=\mathrm{O}(1), \text { as } \quad r \rightarrow \infty,
$$

and so $V$ cannot satisfy (6.1).

Remark 6.1 Since for a solution $(U, V)$ of (5.11) satifying (6.1) we have that, $F_{1}(\infty)<\infty$ and (6.4) holds, then we can use those facts, together with (6.2) and (6.3) to show (as in (6.5) that,

$$
U(r)=-F_{1}(\infty) \ln r+\mathrm{O}(1), \quad V(r)=-F_{2}(\infty) \ln r+\mathrm{O}(1), \quad \text { as } \quad r \rightarrow \infty
$$

and (by recalling (5.5) and (5.6)):

$$
U^{\prime}(r)=-\frac{F_{1}(\infty)}{r}+\mathrm{o}(1), \quad V^{\prime}(r)=-\frac{F_{2}(\infty)}{r}+\mathrm{o}(1), \quad \text { as } \quad r \rightarrow \infty .
$$

More importantly, we observe that not all initial data yield to solutions satisfying (6.1) (or equivalently (6.2)). To see this fact, we use some new estimates specific of solutions of (5.11) satisfying (6.1), which also have the advantage to depend only on $U(0)$.

Lemma 6.2 Let $(U, V)$ be a solution of (5.11) satisfying the integrability condition (6.1), we have:

$$
\begin{aligned}
& r^{2\left(N_{2}+1\right)} \mathrm{e}^{V(r)} \leq 4\left(N_{1}+1\right)\left(N_{2}+1\right), \forall r>0, \\
& r^{2\left(N_{1}+1\right)} \mathrm{e}^{U(r)} \leq C\left(N_{1}, N_{2}\right)\left(1+\mathrm{e}^{U(0)}\right), \forall r>0, \\
& \int_{0}^{\infty} r^{2\left(N_{1}+N_{2}\right)+1} \mathrm{e}^{U(r)+V(r)} \mathrm{d} r \leq C\left(N_{1}, N_{2}\right) \mathrm{e}^{\frac{U(0)}{N_{1}+1}}\left(1+\mathrm{e}^{U(0)}\right)^{\frac{1}{N_{1}+1}},
\end{aligned}
$$

with $C\left(N_{1}, N_{2}\right)$ a suitable positive constant depending only on $N_{1}, N_{2}$.

Proof. In view of (6.1), we know that $v(r)+2 \ln r=V(r)+2\left(N_{2}+1\right) \ln r \rightarrow-\infty$ as $r \rightarrow 0^{+}$ and as $r \rightarrow \infty$. Therefore, $V(r)+2\left(N_{2}+1\right) \ln r$ attains its maximum value, say at $r_{2}>0$, with $F_{2}\left(r_{2}\right)=2\left(N_{2}+1\right)$. By the information provided by Proposition 5.3, we also know that such maximum point is unique and $V\left(r_{2}\right)-2 N_{2} \ln r_{2}=v\left(r_{2}\right)>0$. Thus, by (5.6) and the Pohozaev identity (5.2) applied at $r=r_{2}>0$ we find:

$$
\begin{aligned}
& 2 \int_{0}^{r_{2}} r^{2\left(N_{2}+N_{2}\right)+1} \mathrm{e}^{U(r)+V(r)} \mathrm{d} r+r_{2}^{2\left(N_{1}+1\right)} \mathrm{e}^{U\left(r_{2}\right)}\left(r_{2}^{2 N_{2}} \mathrm{e}^{V\left(r_{2}\right)}-1\right)+r_{2}^{2\left(N_{2}+1\right)} \mathrm{e}^{V\left(r_{2}\right)} \\
& =4\left(N_{1}+1\right)\left(N_{2}+1\right),
\end{aligned}
$$


and we readily deduce that,

$$
r_{2}^{2\left(N_{2}+1\right)} \mathrm{e}^{V\left(r_{2}\right)} \leq 4\left(N_{1}+1\right)\left(N_{2}+1\right)
$$

and (6.8) is established.

Next, let $r_{1}>0$ be the unique maximum point of $u(r)+2 \ln r$. Namely, $U\left(r_{1}\right)+2\left(N_{1}+1\right) \ln r_{1}=$ $\max \left\{U(r)+2\left(N_{1}+1\right) \ln r\right\}$ and so, $F_{1}\left(r_{1}\right)=2\left(N_{1}+1\right)$. Pohozaev's identity (5.2) at $r=r_{1}>0$ gives:

$$
\begin{aligned}
& 2 \int_{0}^{r_{1}} r^{2\left(N_{1}+N_{2}\right)+1} \mathrm{e}^{U(r)+V(r)} \mathrm{d} r+r_{1}^{2\left(N_{1}+N_{2}+1\right)} \mathrm{e}^{U\left(r_{1}\right)+V\left(r_{1}\right)}+r_{1}^{2\left(N_{2}+1\right)} \mathrm{e}^{V\left(r_{1}\right)}-r_{1}^{2\left(N_{1}+1\right)} \mathrm{e}^{U\left(r_{1}\right)} \\
& =4\left(N_{1}+1\right)\left(N_{2}+1\right)
\end{aligned}
$$

or equivalently,

$$
\begin{aligned}
r_{1}^{2\left(N_{1}+1\right)} \mathrm{e}^{U\left(r_{1}\right)}= & 2 \int_{0}^{r_{1}} r^{2\left(N_{1}+N_{2}\right)+1} \mathrm{e}^{U(r)+V(r)} \mathrm{d} r+r_{1}^{2\left(N_{2}+1\right)} \mathrm{e}^{V\left(r_{1}\right)}-4\left(N_{1}+1\right)\left(N_{2}+1\right) \\
& +r_{1}^{2\left(N_{1}+N_{2}+1\right)} \mathrm{e}^{U\left(r_{1}\right)+V\left(r_{1}\right)} .
\end{aligned}
$$

Therefore, if we use (6.14) and the fact that,

$$
\int_{0}^{r_{1}} r^{2\left(N_{1}+N_{2}\right)+1} \mathrm{e}^{U(r)+V(r)} \mathrm{d} r \leq F_{1}\left(r_{1}\right)=2\left(N_{1}+1\right)
$$

we have:

$$
\begin{aligned}
r_{1}^{2\left(N_{1}+1\right)} \mathrm{e}^{U\left(r_{1}\right)} & \leq 2 N_{1}+r_{1}^{2\left(N_{1}+N_{2}+1\right)} \mathrm{e}^{U\left(r_{1}\right)+V\left(r_{1}\right)} \\
& \leq 2 N_{1}+4\left(N_{1}+1\right)\left(N_{2}+1\right) r_{1}^{2 N_{1}} \mathrm{e}^{U\left(r_{1}\right)} .
\end{aligned}
$$

In case there holds: $r_{1}^{2} \leq 8\left(N_{1}+1\right)\left(N_{2}+1\right)$ then,

$$
r_{1}^{2\left(N_{1}+1\right)} \mathrm{e}^{U\left(r_{1}\right)} \leq C\left(N_{1}, N_{2}\right) \mathrm{e}^{U(0)},
$$

with $C\left(N_{1}, N_{2}\right)=\left(8\left(N_{1}+1\right)\left(N_{2}+1\right)\right)^{N_{1}+1}$.

On the other hand, if $r_{1}^{2}>8\left(N_{1}+1\right)\left(N_{2}+1\right)$, then we can use (6.16) to obtain that, $r_{1}^{2\left(N_{1}+1\right)} \mathrm{e}^{U\left(r_{1}\right)} \leq$ $4 N_{1}$, and so in all cases we see that (6.9) holds.

Next we prove (6.10). For any $R>0$, we use (6.8) and (6.9) to find:

$$
\begin{aligned}
\int_{0}^{\infty} t^{2\left(N_{1}+N_{2}\right)+1} \mathrm{e}^{U(t)+V(t)} \mathrm{d} t & =\int_{0}^{R} t^{2\left(N_{1}+N_{2}\right)+1} \mathrm{e}^{U(t)+V(t)} \mathrm{d} t+\int_{R}^{\infty} t^{2\left(N_{1}+N_{2}\right)+1} \mathrm{e}^{U(t)+V(t)} \mathrm{d} t \\
& \leq C\left(N_{1}, N_{2}\right)\left(\int_{0}^{R} t^{2 N_{1}-1} \mathrm{e}^{U(t)} \mathrm{d} t+\int_{R}^{\infty} \frac{1+\mathrm{e}^{U(0)}}{t^{3}}\right) \\
& \leq C\left(N_{1}, N_{2}\right)\left(\mathrm{e}^{U(0)} R^{2 N_{1}}+\frac{1+\mathrm{e}^{U(0)}}{R^{2}}\right)
\end{aligned}
$$

At this point, by minimizing with respect to $R>0$ the right hand side of (6.18) (namely, by taking $R=\left(\frac{1+\mathrm{e}^{U(0)}}{N_{1} \mathrm{e}^{U(0)}}\right)^{\frac{1}{2\left(N_{1}+1\right)}}$ in $\left.(\underline{6.18})\right)$, we obtain:

$$
\int_{0}^{\infty} t^{2\left(N_{1}+N_{2}\right)+1} \mathrm{e}^{U(t)+V(t)} \mathrm{d} t \leq C\left(N_{1}, N_{2}\right) \mathrm{e}^{\frac{U(0)}{N_{1}+1}}\left(1+\mathrm{e}^{U(0)}\right)^{\frac{1}{N_{1}+1}}
$$

and (6.10) is also established. 
Corollary 6.1 There exists $a_{0} \equiv a_{0}\left(N_{1}, N_{2}\right)$ such that if $(U, V)$ is the solution of (5.11) with $U(0)=\alpha_{1}<a_{0}$, then (6.1) (or 6.2) fails.

Proof. Indeed, if by contradiction we suppose that $F_{2}(\infty)>2\left(N_{2}+1\right)$, then we would have: $2\left(N_{2}+1\right) \leq \int_{0}^{\infty} t^{2\left(N_{1}+N_{2}\right)+1} \mathrm{e}^{U(t)+V(t)} \mathrm{d} t$, and by means of the estimates (6.10) we could certainly violate such an inequality by letting $U(0) \rightarrow-\infty$.

Inspired by the shape of the solution constructed in Section 4 , we prove the following:

Theorem 6.1 For any given $L \in \mathbb{R}$ and $\gamma \in\left(2\left(N_{2}+1\right), 2\left(N_{1}+N_{2}+1\right)\right)$ (or $\left.E>0\right)$, there exists $\alpha_{\gamma}=\alpha_{\gamma}(L)$ (or $\alpha=\alpha_{E}(L)$ ) such that, the (unique) solution $(U, V)$ of (5.11) with $(U(0), V(0))=$ $\left(\alpha_{\gamma}, \frac{\left(2 N_{2}+1\right) \alpha_{\gamma}-L}{2 N_{1}+1}\right)$ satisfies:

$$
\gamma=F_{2}(\infty)=\int_{0}^{\infty} r^{2 N_{1}+1} \mathrm{e}^{U(r)}\left(r^{2 N_{2}+1} \mathrm{e}^{V(r)}-1\right) \mathrm{d} r
$$

Similarly, if we fix $\gamma \in\left(2\left(N_{1}+N_{2}+1\right), \infty\right)$, then an analogous statement hold with

$$
\gamma=F_{1}(\infty)=\int_{0}^{\infty} r^{2 N_{2}+1} \mathrm{e}^{V(r)}\left(r^{2 N_{1}+1} \mathrm{e}^{U(r)}+1\right) \mathrm{d} r
$$

While, if $(U(0), V(0))=\left(\alpha_{E}, \frac{\left(2 N_{2}+1\right) \alpha_{E}-L}{2 N_{1}+1}\right)$, then it holds:

$$
N(N-1) \sigma^{3} k \int_{0}^{\infty}\left(t \mathrm{e}^{u(t)}+t \mathrm{e}^{v(t)}\right) \mathrm{d} t=N(N-1) \sigma^{3} k \int_{0}^{\infty}\left(t^{2 N_{1}+1} \mathrm{e}^{U(t)}+t^{2 N_{2}+1} \mathrm{e}^{V(t)}\right)=E
$$

Furthermore, by setting $\beta_{i} \equiv \frac{1}{2} F_{i}(\infty)-N_{i}(i=1,2)$, then $\beta_{1}>N_{2}+1$ and $1<\beta_{2}<N_{1}+1$, and

$$
\begin{aligned}
0<\left(\beta_{1}-1\right)\left(\beta_{2}-1\right) & <\left(N_{1}+1\right)\left(N_{2}+1\right), \\
\beta_{2}\left(\beta_{1}-1\right) & >N_{2}\left(N_{1}+1\right) \\
0<\beta_{1}\left(\beta_{2}-1\right) & <N_{1}\left(N_{2}+1\right) .
\end{aligned}
$$

Remark 6.2 The role of the parameters $\beta_{1}$ and $\beta_{2}$ introduced in Theorem 6.1 can be justified better in terms of the pair $(u(r), v(r)): u(r)=U(r)+2 N_{1} \ln r$ and $v(r)=V(r)+2 N_{2} \ln r$, which defines a radial solution of (3.1) satisfying:

$$
\begin{aligned}
& u(r)=-2 \beta_{1} \ln r+\mathrm{O}(1), \quad v(r)=-2 \beta_{2} \ln r+\mathrm{O}(1), \quad \text { as } \quad r \rightarrow \infty, \\
& u^{\prime}(r)=-\frac{2 \beta_{1}}{r}+\mathrm{o}(1), \quad v^{\prime}(r)=-\frac{2 \beta_{2}}{r}+\mathrm{o}(1), \quad \text { as } \quad r \rightarrow \infty,
\end{aligned}
$$

as we can easily derive from (6.6) and (6.7). In other words, $2 \beta_{1}$ and $2 \beta_{2}$ identify the power of decay at infinity respectively of $\mathrm{e}^{u}$ and $\mathrm{e}^{v}$ (and their derivatives), consistently with (3.2).

To establish Theorem 6.1, we use a blow-up argument together with the information provided by Corollary 6.1, 
Theorem 6.2 For any given $L \in \mathbb{R}$, let $\left(U_{n}(r), V_{n}(r)\right)$ be a sequence of solutions for (5.11) with initial data $\left(U_{n}(0), V_{n}(0)\right)$ satisfying

$$
\left(2 N_{2}+1\right) U_{n}(0)-\left(2 N_{1}+1\right) V_{n}(0) \rightarrow L, \quad \text { as } \quad n \rightarrow \infty .
$$

Let

$$
F_{2, n}(r) \equiv \int_{0}^{r} t^{2\left(N_{1}+N_{2}\right)+1} \mathrm{e}^{U_{n}(t)+V_{n}(t)} \mathrm{d} t-\int_{0}^{r} t^{2 N_{1}+1} \mathrm{e}^{U_{n}(t)} \mathrm{d} t,
$$

the following holds:

$$
\text { if } U_{n}(0) \rightarrow \infty \text {, then } F_{2, n}(\infty) \rightarrow 2\left(N_{1}+N_{2}+1\right) \text {, as } n \rightarrow \infty .
$$

Proof. The proof relies on a blow-up analysis.

In view of Corollary 5.1 we let $0<t_{n} \leq \infty$, be such that $F_{2, n}(t)<0$ for all $t \in\left(0, t_{n}\right)$.

Claim. $0<t_{n} \leq 1$ and so: $F_{2, n}\left(t_{n}\right)=0, V_{n}\left(t_{n}\right)=\max V_{n}$ and $F_{2, n}(t)>0$ for $t>t_{n}$.

We argue by contradiction and suppose that $F_{2, n}(r)<0, \forall r \in[0,1]$. Hence, $V_{n}(r)$ is increasing in $[0,1]$, and we have:

$$
0<V_{n}(r)-V_{n}(0)=-F_{2, n}(r) \ln r+\int_{0}^{r}(\ln t) F_{2, n}^{\prime}(t) \mathrm{d} t, \forall r \in(0,1]
$$

In particular, from (6.31) we find that, $\int_{0}^{r} \ln t F_{2, n}^{\prime}(t) \mathrm{d} t>0, \forall r \in[0,1]$.

Thus, by recalling that $U_{n}(r) \leq U_{n}(0), V_{n}(r)$ is increasing in [0,1], and by using ([6.28), we have:

$$
\begin{aligned}
& \int_{0}^{r}\left(\ln \frac{1}{t}\right) t^{2 N_{1}+1} \mathrm{e}^{U_{n}(t)} \mathrm{d} t \\
& >\int_{0}^{r}\left(\ln \frac{1}{t}\right) t^{2\left(N_{1}+N_{2}\right)+1} \mathrm{e}^{U_{n}(t)+V_{n}(t) \mathrm{d} t} \\
& =\int_{0}^{r}\left(\ln \frac{1}{t}\right) t^{2\left(N_{1}+N_{2}\right)+1} \mathrm{e}^{\frac{2\left(N_{1}+N_{2}+1\right)}{2 N_{1}+1} U_{n}(t)+V_{n}(t)-\frac{2 N_{2}+1}{2 N_{1}+1} U_{n}(t)} \mathrm{d} t \\
& \geq \int_{0}^{r}\left(\ln \frac{1}{t}\right) t^{2\left(N_{1}+N_{2}\right)+1} \mathrm{e}^{\frac{2\left(N_{1}+N_{2}+1\right)}{2 N_{1}+1} U_{n}(t)} \mathrm{e}^{V_{n}(t)-\frac{2 N_{2}+1}{2 N_{1}+1} U_{n}(0)} \mathrm{d} t \\
& \geq \mathrm{e}^{-\frac{L}{2 N_{1}+1}}(1+\mathrm{o}(1)) \int_{0}^{r}\left(\ln \frac{1}{t}\right) t^{2\left(N_{1}+N_{2}\right)+1} \mathrm{e}^{\frac{2\left(N_{1}+N_{2}+1\right)}{2 N_{1}+1} U_{n}(t)} \mathrm{d} t,
\end{aligned}
$$

as $n \rightarrow \infty$. On the other hand, by Hölder's inequality, we get:

$$
\begin{aligned}
& \int_{0}^{r}\left(\ln \frac{1}{t}\right) t^{2 N_{1}+1} \mathrm{e}^{U_{n}(t)} \mathrm{d} t \\
& \leq\left(\int_{0}^{r}\left(\ln \frac{1}{t}\right) t^{2\left(N_{1}+N_{2}\right)+1} \mathrm{e}^{\frac{2\left(N_{1}+N_{2}+1\right)}{2 N_{1}+1} U_{n}(t)} \mathrm{d} t\right)^{\frac{2 N_{1}+1}{2\left(N_{1}+N_{2}+1\right)}}\left(\int_{0}^{r}\left(\ln \frac{1}{t}\right) t^{\frac{2 N_{1}+1}{2 N_{2}+1}} \mathrm{~d} t\right)^{\frac{2 N_{2}+1}{2\left(N_{1}+N_{2}+1\right)}},
\end{aligned}
$$

which we can use together with 6.32 to obtain:

$$
\left(\int_{0}^{r}\left(\ln \frac{1}{t}\right) t^{2 N_{1}+1} \mathrm{e}^{U_{n}(t)} \mathrm{d} t\right)^{\frac{2 N_{2}+1}{2 N_{1}+1}} \leq \mathrm{e}^{\frac{L}{2 N_{1}+1}}(1+\mathrm{o}(1))\left(\int_{0}^{r}\left(\ln \frac{1}{t}\right) t^{\frac{2 N_{1}+1}{2 N_{2}+1}} \mathrm{~d} t\right)^{\frac{2 N_{2}+1}{2 N_{1}+1}}, \forall r \in[0,1],
$$


as $n \rightarrow \infty$. As a consequence, $\forall r \in[0,1]$ and $\forall n \in \mathbb{N}$, we conclude that:

$$
\int_{0}^{r}\left(\ln \frac{1}{t}\right) t^{2\left(N_{1}+N_{2}\right)+1} \mathrm{e}^{U_{n}(t)+V_{n}(t)} \mathrm{d} t<\int_{0}^{r}\left(\ln \frac{1}{t}\right) t^{2 N_{1}+1} \mathrm{e}^{U_{n}(t)} \mathrm{d} t \leq C,
$$

with $C>0$ a suitable constant depending only on $L, N_{1}$ and $N_{2}$. Therefore, from (6.31) and (6.33) we deduce also that,

$$
0<V_{n}(r)-V_{n}(0) \leq C, \forall r \in[0,1]
$$

To obtain the desired contradiction, we use a blow-up analysis for the scaled functions:

$$
\tilde{U}_{n}(r)=U_{n}\left(s_{n} r\right)-U_{n}(0), \quad \tilde{V}_{n}(r)=V_{n}\left(s_{n} r\right)-V_{n}(0), \quad r \in\left[0, \frac{1}{s_{n}}\right]
$$

with

$$
s_{n}=\mathrm{e}^{-\frac{U_{n}(0)+V_{n}(0)}{2\left(N_{1}+N_{2}+1\right)}} .
$$

By means of (6.28), we see that, as $n \rightarrow \infty$,

$$
\begin{aligned}
1 & =s_{n}^{2\left(N_{1}+N_{2}+1\right)} \mathrm{e}^{U_{n}(0)+V_{n}(0)} \\
& =\mathrm{e}^{-\frac{L}{2 N_{1}+1}}(1+\mathrm{o}(1)) s_{n}^{2\left(N_{1}+N_{2}+1\right)} \mathrm{e}^{\frac{2\left(N_{1}+N_{2}+1\right)}{2 N_{1}+1} U_{n}(0)} \\
& =\mathrm{e}^{-\frac{L}{2 N_{1}+1}}(1+\mathrm{o}(1))\left(s_{n}^{2 N_{1}+1} \mathrm{e}^{U_{n}(0)}\right)^{\frac{2\left(N_{1}+N_{2}+1\right)}{2 N_{1}+1}}
\end{aligned}
$$

which implies that,

$$
s_{n}^{2 N_{1}+1} \mathrm{e}^{U_{n}(0)}=\mathrm{O}(1)
$$

and similarly we get,

$$
s_{n}^{2 N_{2}+1} \mathrm{e}^{V_{n}(0)}=\mathrm{O}(1)
$$

Furthermore, $\left(\tilde{U}_{n}, \tilde{V}_{n}\right)$ can be casted as radially symmetric solutions in $D_{n} \equiv\left\{x \in R^{2}|| x \mid<\frac{1}{s_{n}}\right\}$ of the system:

$$
\begin{cases}-\Delta \tilde{U}_{n}=|x|^{2\left(N_{1}+N_{2}\right)} \mathrm{e}^{\tilde{U}_{n}+\tilde{V}_{n}}+\varepsilon_{2, n}|x|^{2 N_{2}} \mathrm{e}^{\tilde{V}_{n}}, & x \in D_{n} \\ -\Delta \tilde{V}_{n}=|x|^{2\left(N_{1}+N_{2}\right)} \mathrm{e}^{\tilde{U}_{n}+\tilde{V}_{n}}-\varepsilon_{1, n}|x|^{2 N_{1}} \mathrm{e}^{\tilde{U}_{n}}, & x \in D_{n}\end{cases}
$$

with

$$
\varepsilon_{1, n} \equiv s_{n}^{2\left(N_{1}+1\right)} \mathrm{e}^{U_{n}(0)} \rightarrow 0, \quad \varepsilon_{2, n} \equiv s_{n}^{2\left(N_{2}+1\right)} \mathrm{e}^{V_{n}(0)} \rightarrow 0, \quad \text { as } n \rightarrow \infty
$$

(see (6.36)-(6.37) ) and in $D_{n}$ the following holds:

$$
\tilde{U}_{n} \leq \tilde{U}_{n}(0)=0, \quad \tilde{V}_{n} \leq C \text { and } \tilde{V}_{n}(0)=0 .
$$

In particular, we have:

$$
-\Delta\left(\tilde{U}_{n}+\tilde{V}_{n}\right)=2|x|^{2\left(N_{1}+N_{2}\right)} \mathrm{e}^{\tilde{U}_{n}+\tilde{V}_{n}}+\varepsilon_{2, n}|x|^{2 N_{2}} \mathrm{e}^{\tilde{V}_{n}}-\varepsilon_{1, n}|x|^{2 N_{1}} \mathrm{e}^{\tilde{U}_{n}}
$$


with $\tilde{U}_{n}(0)+\tilde{V}_{n}(0)=0$ and $\tilde{U}_{n}+\tilde{V}_{n} \leq C$ in $D_{n}$.

Furthermore, by using (6.28) with $U_{n}(0) \rightarrow+\infty$ into the estimates (5.24), or by using estimates similar to those provided in (6.32) (simply by dropping the term $\ln \frac{1}{t}$ ), we find a suitable constant $C>0$ :

$$
\int_{0}^{\frac{1}{s_{n}}} r^{2\left(N_{1}+N_{2}\right)} \mathrm{e}^{\tilde{U}_{n}(r)+\tilde{V}_{n}(r)} \mathrm{d} r=\int_{0}^{1} r^{2\left(N_{1}+N_{2}\right)} \mathrm{e}^{U_{n}(r)+V_{n}(r)} \mathrm{d} r<C .
$$

Therefore, we are in a position to use standard elliptic estimates together with an Harnack type inequality (see e.g. Corollary 5.2.9 in [60], and corresponding application to Liouville type equations discussed therein) and obtain that, along a subsequence, the following holds:

$$
\tilde{U}_{n}+\tilde{V}_{n} \rightarrow \xi, \quad \text { uniformly in } C_{l o c}^{2}\left(\mathbb{R}^{2}\right), \quad \text { as } n \rightarrow \infty
$$

with $\xi$ a radial solution for the Liouville problem:

$$
\left\{\begin{array}{l}
-\Delta \xi=2 r^{2\left(N_{1}+N_{2}\right)} \mathrm{e}^{\xi} \quad \text { in } \quad \mathbb{R}^{2}, \\
\int_{\mathbb{R}^{2}} r^{2\left(N_{1}+N_{2}\right)+1} \mathrm{e}^{\xi(r)} \mathrm{d} r \leq C, \\
\xi(0)=0 .
\end{array}\right.
$$

By the classification result in [13, 14] and [55], we know the explicit expression for $\xi=\xi(r)$ and in particular that it satisfies:

$$
\int_{0}^{\infty} r^{2\left(N_{1}+N_{2}\right)+1} \mathrm{e}^{\xi(r)} \mathrm{d} r=2\left(N_{1}+N_{2}+1\right)
$$

As a consequence, for any $\varepsilon>0$, there exist $R_{\varepsilon}>0$ and $n_{\varepsilon}>0$ such that,

$$
\int_{0}^{R_{\varepsilon}} r^{2\left(N_{1}+N_{2}\right)+1} \mathrm{e}^{\tilde{U}_{n}(r)+\tilde{V}_{n}(r)} \mathrm{d} r \geq 2\left(N_{1}+N_{2}+1\right)-\varepsilon, \quad \forall n \geq n_{\varepsilon} .
$$

On the other hand, since $F_{2, n}(t) \leq 0$ for $0 \leq t \leq 1$, we see that,

$$
\begin{aligned}
\int_{0}^{R_{\varepsilon}} r^{2\left(N_{1}+N_{2}\right)+1} \mathrm{e}^{\tilde{U}_{n}(r)+\tilde{V}_{n}(r)} \mathrm{d} r & =\int_{0}^{s_{n} R_{\varepsilon}} r^{2\left(N_{1}+N_{2}\right)+1} \mathrm{e}^{U_{n}(r)+V_{n}(r)} \mathrm{d} r \\
& <\int_{0}^{s_{n} R_{\varepsilon}} r^{2 N_{1}+1} \mathrm{e}^{U_{n}(r)} \mathrm{d} r \\
& \leq \frac{\mathrm{e}^{U_{n}(0)}}{2\left(N_{1}+1\right)} s_{n}^{2\left(N_{1}+1\right)} R_{\varepsilon}^{2\left(N_{1}+1\right)} \rightarrow 0 \text { as } n \rightarrow \infty
\end{aligned}
$$

which leads to the desired contradiction, and the Claim is established.

Therefore, we can use the estimates (6.31) for $r=t_{n}$, and as above deduce that,

$$
0<V_{n}\left(t_{n}\right)-V_{n}(0)=\int_{0}^{t_{n}}(\ln t) F_{2, n}^{\prime}(t) \mathrm{d} t \leq C .
$$

Hence, for the scaled functions $\left(\tilde{U}_{n}, \tilde{V}_{n}\right)$ now we can claim that,

$$
\begin{array}{r}
\tilde{U}_{n}(r) \leq \tilde{U}_{n}(0)=0, \quad \tilde{V}_{n}(r) \leq V_{n}\left(\frac{t_{n}}{s_{n}}\right) \leq C, \tilde{V}_{n}(0)=0, \\
\int_{0}^{\infty} r^{2\left(N_{1}+N_{2}\right)+1} \mathrm{e}^{U_{n}+V_{n}} \leq C, \quad \int_{0}^{\infty} r^{2 N_{1}+1} \mathrm{e}^{U_{n}} \leq C,
\end{array}
$$


where the estimates (6.50) hold with a constant $C>0$ depending only on $N_{1}, N_{2}$ and $L$, and they can be derived by using (6.28) with $U_{n}(0) \rightarrow \infty$, into the estimates (5.21) and (5.25), as follows:

$$
\begin{gathered}
\int_{0}^{\infty} t^{2 N_{1}+1} \mathrm{e}^{U_{n}(t)} \mathrm{d} t \leq 4\left(N_{1}+1\right)+2 \mathrm{e}^{U_{n}(0)-\frac{N_{1}+1}{N_{2}} V_{n}(0)}=4\left(N_{1}+1\right)+\mathrm{o}(1) \\
\int_{0}^{\infty} t^{2\left(N_{1}+N_{2}\right)+1} \mathrm{e}^{U_{n}(t)+V_{n}(t)} \mathrm{d} t \leq 6\left(N_{1}+1\right)+2 N_{2}+2 \mathrm{e}^{U_{n}(0)-\frac{N_{1}+1}{N_{2}} V_{n}(0)} \\
=6\left(N_{1}+1\right)+2 N_{2}+\mathrm{o}(1)
\end{gathered}
$$

as $n \rightarrow \infty$.

As above, we can show that:

$$
0<\frac{t_{n}}{s_{n}}<C, \text { for some } C>0 .
$$

Indeed, by assuming that (along a subsequence), $\frac{t_{n}}{s_{n}} \rightarrow \infty$, then as before, we can carry out a blow-up argument in $\left[0, \frac{t_{n}}{s_{n}}\right)$ to get a contradiction.

Therefore, in view of (6.49), (6.50), (6.53) as above by well-known elliptic and Harnack estimates, we obtain that, along a subsequence,

$$
\tilde{U}_{n} \rightarrow U, \quad \tilde{V}_{n} \rightarrow V \quad \text { in } \quad C_{l o c}^{2}\left(\mathbb{R}^{2}\right)
$$

with $(U, V)$ a radial solution of the problem:

$$
\left\{\begin{array}{l}
-\Delta U=|x|^{2\left(N_{1}+N_{2}\right)} \mathrm{e}^{U+V} \quad \text { in } \quad \mathbb{R}^{2}, \\
-\Delta V=|x|^{2\left(N_{1}+N_{2}\right)} \mathrm{e}^{U+V} \quad \text { in } \quad \mathbb{R}^{2}, \\
\int_{\mathbb{R}^{2}}|x|^{2\left(N_{1}+N_{2}\right)} \mathrm{e}^{U+V} \mathrm{~d} x<\infty, \quad U(0)=0=V(0) .
\end{array}\right.
$$

In particular, $U+V$ defines a radially symmetric solution of the Liouville problem:

$$
\left\{\begin{array}{l}
-\Delta(U+V)=2|x|^{2\left(N_{1}+N_{2}\right)} \mathrm{e}^{U+V} \quad \text { in } \quad \mathbb{R}^{2}, \\
\int_{\mathbb{R}^{2}}|x|^{2\left(N_{1}+N_{2}\right)} \mathrm{e}^{U+V} \mathrm{~d} x<\infty
\end{array}\right.
$$

which satisfies: $\max (U+V)=U(0)+V(0)=0$.

Therefore, by the classification result of [55], we get the following explicit expression:

$$
U(r)+V(r)=\ln \left(\frac{1}{\left(1+\frac{r^{2\left(N_{1}+N_{2}+1\right)}}{4\left(N_{1}+N_{2}+1\right)^{2}}\right)^{2}}\right),
$$

and

$$
\int_{0}^{\infty} r^{2\left(N_{1}+N_{2}\right)+1} \mathrm{e}^{U(r)+V(r)} \mathrm{d} r=2\left(N_{1}+N_{2}+1\right) .
$$

In addition, by using (the radial expression of) (6.55) and (6.57) we readily check that $U$ and $V$ admit the same logarithmic growth at $\infty$. As a consequence, $U-V$ defines a bounded harmonic function in $\mathbb{R}^{2}$, which vanishes at the origin, in other words $U-V \equiv 0$, and we obtain:

$$
U(r)=\ln \left(\frac{1}{1+\frac{r^{2\left(N_{1}+N_{2}+1\right)}}{4\left(N_{1}+N_{2}+1\right)^{2}}}\right)=V(r), \quad \forall r \geq 0 .
$$


In particular, we observe that: $\frac{t_{n}}{s_{n}} \rightarrow 0$.

We can use those information to see that, for any $0<\varepsilon<\frac{1}{4} \min \left\{N_{1}, N_{2}\right\}$ sufficiently small, there exist $n_{\varepsilon} \in \mathbb{N}$ and $R_{\varepsilon} \gg 1$ such that, for $n \geq n_{\varepsilon}$ and $r \geq R_{\varepsilon}$, we have:

$$
\begin{aligned}
\tilde{F}_{1, n}(r) & \equiv \int_{0}^{r} t^{2\left(N_{1}+N_{2}\right)+1} \mathrm{e}^{\tilde{U}_{n}(t)+\tilde{V}_{n}(t)} \mathrm{d} t+\varepsilon_{2, n} \int_{0}^{r} t^{2 N_{2}} \mathrm{e}^{\tilde{V}_{n}(t)} \mathrm{d} t \\
& \geq \int_{0}^{r} t^{2\left(N_{1}+N_{2}\right)+1} \mathrm{e}^{\tilde{U}_{n}(t)+\tilde{V}_{n}(t)} \mathrm{d} t>2\left(N_{1}+N_{2}+1-\varepsilon\right) .
\end{aligned}
$$

As a consequence, we can check that for $n \geq n_{\varepsilon}$, the function:

$$
r^{2\left(N_{1}+1+\varepsilon\right)} \mathrm{e}^{\tilde{U}_{n}(r)} \quad \text { is decreasing } \quad \forall r \geq R_{\varepsilon},
$$

indeed:

$$
\begin{aligned}
\frac{\mathrm{d}}{\mathrm{d} r}\left(r^{2\left(N_{1}+1+\varepsilon\right)} \mathrm{e}^{\tilde{U}_{n}(r)}\right) & =r^{2\left(N_{1}+\varepsilon\right)+1} \mathrm{e}^{\tilde{U}_{n}(r)}\left[2\left(N_{1}+1+\varepsilon\right)-\tilde{F}_{1, n}(r)\right] \\
& \leq r^{2\left(N_{1}+\varepsilon\right)+1} \mathrm{e}^{\tilde{U}_{n}(r)}\left[2\left(N_{1}+1+\varepsilon\right)-2\left(N_{1}+N_{2}+1-\varepsilon\right)\right] \\
& =-2 r^{2\left(N_{1}+\varepsilon\right)+1} \mathrm{e}^{\tilde{U}_{n}(r)}\left(N_{2}-2 \varepsilon\right)<0, \quad \forall r \geq R_{\varepsilon} .
\end{aligned}
$$

Therefore, for $r \geq R_{\varepsilon}$ we find:

$$
r^{2\left(N_{1}+1+\varepsilon\right)} \mathrm{e}^{\tilde{U}_{n}(r)} \leq R_{\varepsilon}^{2\left(N_{1}+1+\varepsilon\right)} \mathrm{e}^{\tilde{U}_{n}\left(R_{\varepsilon}\right)} \rightarrow \frac{R_{\varepsilon}^{2\left(N_{1}+1+\varepsilon\right)}}{1+\frac{R_{\varepsilon}^{\left(N_{1}+N_{2}+1\right)}}{4\left(N_{1}+N_{2}+1\right)^{2}}}, \quad \text { as } \quad n \rightarrow \infty ;
$$

and so, for a suitably constant $C_{\varepsilon}>0$, we have:

$$
r^{2\left(N_{1}+1+\varepsilon\right)} \mathrm{e}^{\tilde{U}_{n}(r)} \leq C_{\varepsilon}, \quad \forall r \geq R_{\varepsilon}, \forall n \geq n_{\varepsilon} .
$$

As a consequence,

$$
\int_{0}^{\infty} r^{2 N_{1}+1} \mathrm{e}^{\tilde{U}_{n}(r)} \mathrm{d} r<C
$$

for suitable $C>0$, and we conclude the important fact,

$$
\int_{0}^{\infty} r^{2 N_{1}+1} \mathrm{e}^{U_{n}(r)} \mathrm{d} r=\varepsilon_{1, n} \int_{0}^{\infty} r^{2 N_{1}+1} \mathrm{e}^{\tilde{U}_{n}(r)} \rightarrow 0, \text { as } n \rightarrow \infty .
$$

Similarly, we can check that (by taking $n_{\varepsilon}$ larger if necessary)

$$
r^{2\left(N_{2}+1+\varepsilon\right)} \mathrm{e}^{\tilde{V}_{n}(r)} \quad \text { is decreasing } \quad \forall r \geq R_{\varepsilon}, \forall n \geq n_{\varepsilon},
$$

simply by computing:

$$
\begin{aligned}
& \frac{\mathrm{d}}{\mathrm{d} r}\left(r^{2\left(N_{2}+1+\varepsilon\right)} \mathrm{e}^{\tilde{V}_{n}(r)}\right) \\
& =r^{2\left(N_{2}+\varepsilon\right)+1} \mathrm{e}^{\tilde{V}_{n}(r)}\left(2\left(N_{2}+1+\varepsilon\right)-\tilde{F}_{2, n}(r)\right) \\
& \equiv r^{2\left(N_{2}+\varepsilon\right)+1} \mathrm{e}^{\tilde{V}_{n}(r)}\left[2\left(N_{2}+1+\varepsilon\right)-\int_{0}^{r} t^{2\left(N_{1}+N_{2}\right)} \mathrm{e}^{\tilde{U}_{n}(t)+\tilde{V}_{n}(t)} \mathrm{d} r+\varepsilon_{1, n} \int_{0}^{r} t^{2 N_{1}} \mathrm{e}^{\tilde{U}_{n}(t)} \mathrm{d} t\right] \\
& \leq r^{2\left(N_{2}+\varepsilon\right)+1} \mathrm{e}^{\tilde{V}_{n}(r)}\left[2\left(N_{2}+1+\varepsilon\right)-2\left(N_{1}+N_{2}+1-\varepsilon\right)+\mathrm{o}(1)\right] \\
& \leq-2 r^{2\left(N_{2}+\varepsilon\right)+1} \mathrm{e}^{\tilde{V}_{n}(r)}\left(N_{1}-2 \varepsilon+\mathrm{o}(1)\right)<0, \quad \text { as } \quad n \rightarrow \infty .
\end{aligned}
$$


Therefore, exactly as above we can show that,

$$
\int_{0}^{\infty} r^{2 N_{2}+1} \mathrm{e}^{V_{n}(r)} \mathrm{d} r=\varepsilon_{2, n} \int_{0}^{\infty} r^{2 N_{2}+1} \mathrm{e}^{\tilde{V}_{n}(r)} \mathrm{d} r \rightarrow 0 \text { as } n \rightarrow \infty .
$$

With this information, we can finally show that (by taking $n_{\varepsilon}$ larger if necessary)

$$
r^{2\left(N_{1}+N_{2}+1+\varepsilon\right)} \mathrm{e}^{\tilde{U}_{n}+\tilde{V}_{n}} \quad \text { is decreasing, } \forall r \geq R_{\varepsilon}, \forall n \geq n_{\varepsilon},
$$

as we have:

$$
\begin{aligned}
& \frac{\mathrm{d}}{\mathrm{d} r}\left[r^{2\left(N_{1}+N_{2}+1+\varepsilon\right)} \mathrm{e}^{\left.\tilde{U}_{n}(r)+\tilde{V}_{n}(r)\right]}\right. \\
& =r^{2\left(N_{1}+N_{2}+\varepsilon\right)+1} \mathrm{e}^{\tilde{U}_{n}(r)+\tilde{V}_{n}(r)}\left[2\left(N_{1}+N_{2}+1+\varepsilon\right)-\tilde{F}_{1, n}(r)-\tilde{F}_{2, n}(r)\right] \\
& \leq r^{2\left(N_{1}+N_{2}+\varepsilon\right)+1} \mathrm{e}^{\tilde{U}_{n}(r)+\tilde{V}_{n}(r)}\left[2\left(N_{1}+N_{2}+1+\varepsilon\right)-2 \int_{0}^{r} t^{2\left(N_{1}+N_{2}\right)+1} \mathrm{e}^{\tilde{U}_{n}(t)+\tilde{V}_{n}(t)}+\mathrm{o}(1)\right] \\
& \leq-2 r^{2\left(N_{1}+N_{2}+\varepsilon\right)+1} \mathrm{e}^{\tilde{U}_{n}(r)+\tilde{V}_{n}(r)}\left(N_{1}+N_{2}-3 \varepsilon+\mathrm{o}(1)\right)<0, \quad \text { as } n \rightarrow \infty .
\end{aligned}
$$

So, we can use the convergence: $\left(\tilde{U}_{n}+\tilde{V}_{n}\right)\left(R_{\varepsilon}\right) \rightarrow(U+V)\left(R_{\varepsilon}\right)$ as $n \rightarrow \infty$, to obtain as above a suitable constant $C_{\varepsilon}>0$ such that, for $n \geq n_{\varepsilon}$ and $r \geq R_{\varepsilon}$,

$$
r^{2\left(N_{1}+N_{2}\right)+1} \mathrm{e}^{\tilde{U}_{n}+\tilde{V}_{n}} \leq \frac{C_{\varepsilon}}{r^{1+2 \varepsilon}},
$$

which allows us to conclude that, as $n \rightarrow \infty$ :

$$
\int_{0}^{\infty} r^{2\left(N_{1}+N_{2}\right)+1} \mathrm{e}^{\tilde{U}_{n}(r)+\tilde{V}_{n}(r)} \mathrm{d} r \rightarrow \int_{0}^{\infty} r^{2\left(N_{1}+N_{2}\right)+1} \mathrm{e}^{U(r)+V(r)} \mathrm{d} r=2\left(N_{1}+N_{2}+1\right) .
$$

Clearly, (6.65), (6.68) and (6.72) imply that:

$$
F_{2, n}(\infty) \rightarrow 2\left(N_{1}+N_{2}+1\right), \quad \text { as } \quad n \rightarrow \infty,
$$

and (6.30) is established.

\section{The proof of Theorem 6.1.}

In view of Theorem 6.2, for any fixed constant $L \in \mathbb{R}$, we take the initial data in the form:

$$
\left(\alpha_{1}, \alpha_{2}\right) \equiv\left(\alpha, \frac{\left(2 N_{2}+1\right) \alpha-L}{2 N_{1}+1}\right), \quad \alpha \in \mathbb{R},
$$

and denote by $F_{2, \alpha}(\infty)$ the expression in (5.10) corresponding to the unique solution $\left(U_{\alpha}, V_{\alpha}\right)$ of (5.11) with initial data specified in (6.74). To simplify notations, we do not emphasize the dependence on $L$.

By virtue of Corollary 6.1, there exists $a_{0}$ such that, for any $\alpha<a_{0}$,

$$
\left.F_{2, \alpha}(\infty)<2\left(N_{2}+1\right) \quad \text { (or equivalently } F_{1, \alpha}(\infty)=\infty\right) .
$$

On the other hand, by Theorem 6.2, we know that,

$$
F_{2, \alpha}(\infty) \rightarrow 2\left(N_{1}+N_{2}+1\right), \quad \text { as } \quad \alpha \rightarrow \infty .
$$


Therefore, by the continuity of $F_{2, \alpha}(\infty)$ (or $\left.F_{1, \alpha}(\infty)\right)$ with respect to $\alpha$, for any $\gamma \in\left(2\left(N_{2}+\right.\right.$ 1), $\left.2\left(N_{1}+N_{2}+1\right)\right)$, there exists (at least) a parameter $\alpha_{\gamma}=\alpha_{\gamma}(L)$ such that the Cauchy problem (5.11) with initial data given by (6.74) with $\alpha=\alpha_{\gamma}$ admits a solution $\left(U_{\alpha_{\gamma}}, V_{\alpha_{\gamma}}\right)$ satisfies the integrability condition (6.1) and $F_{2, \alpha_{\gamma}}(\infty)=\gamma$. In other words, we get a radial solution of (3.1) in the form $\left(u_{\alpha_{\gamma}}, v_{\alpha_{\gamma}}\right)=\left(U_{\alpha_{\gamma}}+2 N_{1} \ln r, V_{\alpha_{\gamma}}+2 N_{2} \ln r\right)$.

Since for $L_{1} \neq L_{2}$ the corresponding set of initial data satisfies:

$$
\left(\alpha_{\gamma}\left(L_{1}\right), \frac{\left(2 N_{2}+1\right) \alpha_{\gamma}\left(L_{1}\right)-L_{1}}{2 N_{1}+1}\right) \neq\left(\alpha_{\gamma}\left(L_{2}\right), \frac{\left(2 N_{2}+1\right) \alpha_{\gamma}\left(L_{2}\right)-L_{2}}{2 N_{1}+1}\right)
$$

in this way we have obtained a 1-parameter family of different solutions of (3.1), all satisfying: $F_{2}(\infty)=\gamma \in\left(2\left(N_{2}+1\right), 2\left(N_{1}+N_{2}+1\right)\right)$.

Furthermore, from the version (3.10) of the Pohozaev identity, it follows that: as $\alpha \rightarrow \infty$, if $F_{2, \alpha}(\infty) \rightarrow 2\left(N_{1}+N_{2}+1\right)$, then $\int_{0}^{\infty} r^{2 N_{1}+1} \mathrm{e}^{U_{\alpha}} \mathrm{d} r \rightarrow 0, \int_{0}^{\infty} r^{2 N_{2}+1} \mathrm{e}^{V_{\alpha}} \mathrm{d} r \rightarrow 0$ and $F_{1, \alpha}(\infty) \rightarrow$ $2\left(N_{1}+N_{2}+1\right)$.

As a consequence, if we fix $\gamma \in\left(2\left(N_{1}+N_{2}+1\right), \infty\right)$, we obtain the same conclusion as above with suitable $\alpha_{\gamma} \in \mathbb{R}: F_{1, \alpha_{\gamma}}(\infty)=\gamma$. Similarly, if we set:

$$
E_{\alpha}=N(N-1) \sigma^{3} k \int_{0}^{\infty}\left(r^{2 N_{1}+1} \mathrm{e}^{U_{\alpha}}+r^{2 N_{2}+1} \mathrm{e}^{V_{\alpha}}\right) \mathrm{d} r
$$

then we see that: $E_{\alpha} \rightarrow 0$ as $\alpha \rightarrow \infty$, while $E_{\alpha}=\infty$ for $\alpha<a_{0}$, and for every $E>0$ the above conclusion follows with suitable $\alpha_{E} \in \mathbb{R}: E_{\alpha_{E}}=E$, and the existence part of Theorem 6.1 is established.

Finally, by virtue of (5.39) and (6.2), we check that: $\beta_{1} \equiv \frac{1}{2} F_{1}(\infty)-N_{1}>N_{2}+1$ and $1<\beta_{2} \equiv \frac{1}{2} F_{2}(\infty)-N_{2}<N_{1}+1$. Moreover, to obtain (6.23)-(6.25), we recall that,

$$
\lim _{r \rightarrow \infty} r u^{\prime}(r)=-2 \beta_{1}, \quad \lim _{r \rightarrow \infty} r v^{\prime}(r)=-2 \beta_{2},
$$

and so, by taking the limit $r \rightarrow \infty$ in (5.2)-(5.4), we have:

$$
\begin{aligned}
& 2 \int_{0}^{\infty} t \mathrm{e}^{u(t)+v(t)} \mathrm{d} t=4\left(N_{1}+1\right)\left(N_{2}+1\right)-4\left(\beta_{1}-1\right)\left(\beta_{2}-1\right), \\
& 2 \int_{0}^{\infty} t \mathrm{e}^{u(t)} \mathrm{d} t=4 N_{1}\left(N_{2}+1\right)-4 \beta_{1}\left(\beta_{2}-1\right), \\
& 2 \int_{0}^{\infty} t \mathrm{e}^{v(t)} \mathrm{d} t=4 \beta_{2}\left(\beta_{1}-1\right)-4 N_{2}\left(N_{1}+1\right),
\end{aligned}
$$

from which (6.23) - 6.25) easily follow and the proof of Theorem 6.1 is complete.

\section{The proof of Theorem 2.2 and Theorem 2.3}

By virtue of the results established above about problem (3.1), we can easily obtain the proof of Theorem 2.2 and Theorem 2.3 .

Indeed, by recalling (2.48)-(2.49) (considered with the lower sign) and (2.71), we see that, via 
(2.67)-(2.70), the following holds for the fluxes:

$$
\begin{aligned}
& \frac{1}{2 \pi} \Phi_{1}=\int_{0}^{\infty} f_{12}^{(1)}(r) r \mathrm{~d} r=\frac{8 \pi^{2}}{k^{2}} \int_{0}^{\infty}\left|\phi_{2}(r)\right|^{2}\left(\left|\phi_{1}(r)\right|^{2}+c^{2}\right) r \mathrm{~d} r \\
& =2 \sigma^{2} \int_{0}^{\infty} \mathrm{e}^{v(r)}\left(\mathrm{e}^{u(r)}+1\right) r \mathrm{~d} r=\frac{1}{2} F_{1}(\infty), \\
& \frac{1}{2 \pi} \Phi_{2}=\int_{0}^{\infty} f_{12}^{(2)}(r) r \mathrm{~d} r=\frac{8 \pi^{2}}{k^{2}} \int_{0}^{\infty}\left|\phi_{1}(r)\right|^{2}\left(\left|\phi_{2}(r)\right|^{2}-c^{2}\right) r \mathrm{~d} r \\
& =2 \sigma^{2} \int_{0}^{\infty} \mathrm{e}^{u(r)}\left(\mathrm{e}^{v(r)}-1\right) r \mathrm{~d} r=\frac{1}{2} F_{2}(\infty),
\end{aligned}
$$

where the last identity in (6.82) and (6.83) takes into account the scaling (2.79) and (2.75). Similarly, for the total energy we have:

$$
E=N(N-1) \sigma^{3} k \int_{0}^{\infty}\left(\mathrm{e}^{u(r)}+\mathrm{e}^{v(r)}\right) r \mathrm{~d} r
$$

At this point, still by keeping in mind (2.67)-(2.72), we see that Theorem 2.2 and Theorem 2.3 follow directly by (3.12) (or (5.39) ), Theorem 6.1 and (6.26)-(6.27).

\section{References}

[1] O. Aharony, O. Bergman, D. L. Jafferis, and J. Maldacena, $\mathcal{N}=6$ superconformal Chern-Simons-matter theories, M2-branes and their gravity duals, J. High Energy Phys. 10 (2008) 091.

[2] L. Anderson and J. G. Russo, ABJM theory with mass and FI deformations and quantum phase transitions, J. High Energy Phys. 05 (2015) 064.

[3] R. Auzzi and S. P. Kumar, Non-Abelian vortices at weak and strong coupling in mass deformed ABJM theory, J. High Energy Phys. 10 (2009) 071.

[4] J. Bagger and N. Lambert, Modeling multiple M2's, Phys. Rev. D 75 (2007) 045020.

[5] J. Bagger and N. Lambert, Gauge symmetry and supersymmetry of multiple M2-branes, Phys. Rev. D 77 (2008) 065008.

[6] J. Bagger and N. Lambert, Comments on multiple M2-branes, J. High Energy Phys. 0802 (2008) 105.

[7] Y. Bea, N. Jokela, M. Lippert, A. V. Ramallo, and D. Zoakos, Flux and Hall states in ABJM with dynamical flavors, J. High Energy Phys. 03 (2015) 009.

[8] E. B. Bogomol'nyi, The stability of classical solutions, Sov. J. Nucl. Phys. 24 (1976) 449-454.

[9] D. Chae and O. Y. Imanuvilov, The existence of non-topological multivortex solutions in the relativistic self-dual Chern-Simons theory, Commun. Math. Phys. 215 (2000) 119-142.

[10] D. Chae and G. Tarantello, On planar self-dual electroweak vortices, Ann. Inst. H. Poincaré Anal. Non Linéaire 21 (2004) 187-207.

[11] D. Chae and G. Tarantello, Selfgravitating electroweak strings, J. Diff. Equat. 213 (2005) 146-170.

[12] S. Chen, X. Han, G. Lozano, and F. A. Schaposnik, Existence theorems for non-Abelian Chern-SimonsHiggs vortices with flavor, J. Diff. Equat., 259 (2015) 2458-2498.

[13] W. Chen and C. Li, Classification of solutions of some nonlinear elliptic equations, Duke Math. J. 63 (1991) 615-622. 
[14] W. Chen and C. Li, Qualitative properties of solutions to some nonlinear elliptic equations in $\mathbb{R}^{2}$, Duke Math. J. 71 (1993) 427-439.

[15] S. Chen and Y. Yang, Existence of multiple vortices in supersymmetric gauge field theory, Proc. R. Soc. A 468 (2012) 3923-3946.

[16] M. N. Chernodub, J. Van Doorsselaere, and H. Verschelde, Electromagnetically superconducting phase of the vacuum in a strong magnetic field: structure of superconductor and superfluid vortex lattices in the ground state, Phys. Rev. D 85 (2012) 045002.

[17] H. J. de Vega and F. A. Schaposnik, Electrically charged vortices in nonabelian gauge theories with Chern-Simons term, Phys. Rev. Lett. 56 (1986) 2564

[18] N. Drukker, M. Mariño and P. Putrov, From weak to strong coupling in ABJM theory, Commun. Math. Phys. 306 (2011) 511-563.

[19] G. Dunne, Self-Dual Chern-Simons Theories, Lecture Notes in Physics, vol. m 36, Springer, Berlin, 1995.

[20] J. Fröhlich, The fractional quantum Hall effect, Chern-Simons theory, and integral lattices, Proc. Internat. Congr. Math., pp. 75-105, Birkhäuser, Basel, 1995.

[21] J. Gomis, D. Rodriguez-Gomez, M. Van Raamsdonk and H. Verlinde, A massive study of M2-brane proposals, J. High Energy Phys. 09 (2008) 113.

[22] A. Gustafson, I. M. Sigal, and T. Tzaneteas, Statics and dynamics of magnetic vortices and of NielsenOlesen (Nambu) strings, J. Math. Phys. 51 (2010) 015217.

[23] A. Gustavsson, Algebraic structures on parallel M2-branes, Nucl. Phys. B 811 (2009) 66-76.

[24] X. Han, C.-S. Lin, G. Tarantello and Y. Yang, Chern-Simons vortices in the Gudnason model. J. Funct. Anal. 267, 678-726 (2014).

[25] X. Han, C.-S. Lin, and Y. Yang, Resolution of Chern-Simons-Higgs Vortex Equations, Commun. Math. Phys. 343 (2016) 701-724.

[26] X. Han and G. Tarantello, Doubly periodic self-dual vortices in a relativistic non-Abelian Chern-Simons model, Calc. Var. PDE 49 (2014) 1149-1176.

[27] X. Han, Y. Yang, Existence theorems for vortices in the Aharony-Bergman-Jafferis-Maldacena model, Commun. Math. Phys. 333 (2015) 229-259.

[28] J. Hong, Y. Kim and P.-Y. Pac, Multivortex solutions of the Abelian Chern-Simons-Higgs theory, Phys. Rev. Lett. 64 (1990) 2330-2333.

[29] Y.-H. Hyun, Y. Kim, O-Kab Kwon, and D.D. Tolla, Abelian projections of the mass-deformed ABJM theory and weakly curved dual geometry, Phys. Rev. D 87 (2013) 085011.

[30] A. Jaffe and C. H. Taubes, Vortices and Monopoles, Birkhäuser, Boston, 1980.

[31] R. Jackiw and E. J. Weinberg, Self-dual Chern-Simons vortices, Phys. Rev. Lett. 64 (1990) 2334-2337.

[32] A. Jaffe and C. H. Taubes, Vortices and Monopoles, Birkhäuser, Boston, 1980.

[33] D. I. Khomskii and A. Freimuth, Charged vortices in high temperature superconductors, Phys. Rev. Lett. 75 (1995) 1384-1386.

[34] C. Kim, Y. Kim, O. K. Kwon, and H. Nakajima, Vortex-type half-BPS solitons in ABJM theory, Phys. Rev. D 80 (2009) 045013.

[35] C. Kim, Vortex-type solutions in ABJM theory, J. Phys. (conf. series) 343 (2012) 012057. 
[36] K.-M. Lee, Self-dual nonabelian Chern-Simons solitons, Phys. Rev. Lett. 66 (1991) 553-555.

[37] C.-K. Lee, K.-M. Lee and E.J. Weinberg, Supersymmetry and self-dual Chern-Simons systems, Phys. Lett. B 243 (1990) 105-108.

[38] E. H. Lieb and Y. Yang, Non-Abelian vortices in supersymmetric gauge field theory via direct methods, Commun. Math. Phys. 313 (2012) 445-478.

[39] C.-S. Lin, A. C. Ponce, and Y. Yang, A system of elliptic equations arising in Chern-Simons field theory, J. Funct. Anal. 247 (2007) 289-350.

[40] C. Lopez-Arcos, H. Nastase, F. Rojas, and J. Murugan, Conductivity in the gravity dual to massive ABJM and the membrane paradigm, J. High Energy Phys. 01 (2014) 036.

[41] Y. Matsuda, K. Nozakib, and K. Kumagaib, Charged vortices in high temperature superconductors probed by nuclear magnetic resonance, J. Phys. Chem. Solids 63 (2002) 1061-1063.

[42] A. Mohammed, J. Murugan, and H. Nastase, Towards a realization of the Condensed-Matter-Gravity correspondence in string theory via consistent abelian truncation of the Aharony-Bergman-JafferisMaldacena model, Phys. Rev. Lett. 109 (2012) 181601.

[43] A. Mohammed, J. Murugan, and H. Nastase, Abelian-Higgs and vortices from ABJM: towards a string realization of AdS/CMT, J. High Energy Phys. 11 (2012) 073.

[44] S. Moriyama and T. Suyama, Instanton effects in orientifold ABJM theory, J. High Energy Phys. 03 (2016) 034.

[45] J. Murugan and H. Nastase, On Abelianizations of the ABJM Model and Applications to Condensed Matter, Brazilian Journal of Physics 45 (2015) 481-492.

[46] J. Murugan, H. Nastase, and N. Rughoonauth, J.P. Shock, Particle-vortex and Maxwell duality in the $A d S_{4} \times C P^{3} / \mathrm{ABJM}$ correspondence, J. High Energy Phys. 10 (2014) 051.

[47] H. Nastase and C. Papageorgakis, Dimensional reduction of the ABJM model, J. High Energy Phys. 03 (2011) 094 .

[48] H. Nastase and C. Papageorgakis, and S. Ramgoolam, The fuzzy $S^{2}$ structure of $M 2-M 5$ systems in ABJM membrane theories, J. High Energy Phys. 05 (2009) 123.

[49] H. Nastase and C. Papageorgakis, Bifundamental fuzzy 2-sphere and fuzzy Killing spinors, Symmetry, Integrability and Geometry: Methods and Applications 6 (2010) 058.

[50] H. Nielsen and P. Olesen, Vortex-line models for dual strings, Nucl. Phys. B 61 (1973) 45-61.

[51] L. Nirenberg, Topics in Nonlinear Analysis, in: Courant Lecture Notes in Math., American Mathematical Society, 2001.

[52] T. Nosaka, K. Shimizu, and S. Terashima, Large $N$ behavior of mass deformed ABJM theory, J. High Energy Phys. 03 (2016) 063.

[53] S. Palmer C. Sämann, The ABJM model is a higher gauge theory, Int. J. Geom. Methods Mod. Phys. 11 (2014) 1450075.

[54] S. K. Paul and A. Khare, Charged vortices in Abelian Higgs model with Chern-Simons term, Phys. Lett. B 174 (1986) 420-422.

[55] J. Prajapat and G. Tarantello, On a class of elliptic problems in $\mathbb{R}^{2}$ : symmetry and uniqueness results, Proc. Roy. Soc. Edinburgh Sect. A 131 (2001) 967-985.

[56] M. K. Prasad and C. M. Sommerfield, Exact classical solutions for the 't Hooft monopole and the Julia-Zee dyon, Phys. Rev. Lett. 35 (1975) 760-762. 
[57] J. H. Schwarz, Superconformal Chern-Simons theories, J. High Energy Phys. 11 (2004) 078.

[58] S. I. Shevchenko, Charged vortices in superfluid systems with pairing of spatially separated carriers, Phys. Rev. B 67 (2003) 214515.

[59] J. B. Sokoloff, Charged vortex excitations in quantum Hall systems, Phys. Rev. B 31 (1985) 1924-1928.

[60] G. Tarantello, Self-Dual Gauge Field Vortices, an Analytic Approach, Progress in Nonlinear Differential Equations and Their Applications 72, Birkhäuser, Boston, Basel, Berlin, 2008.

[61] S. Terashima, On M5-branes in $\mathcal{N}=6$ membrane action, J. High Energy Phys. 08 (2008) 080.

[62] F. Wilczek, Fractional Statistics and Anyonic Superconductivity, World Scientific, Singapore 1990.

[63] Y. Yang, Solitons in Field Theory and Nonlinear Analysis, Springer, New York, 2001. 